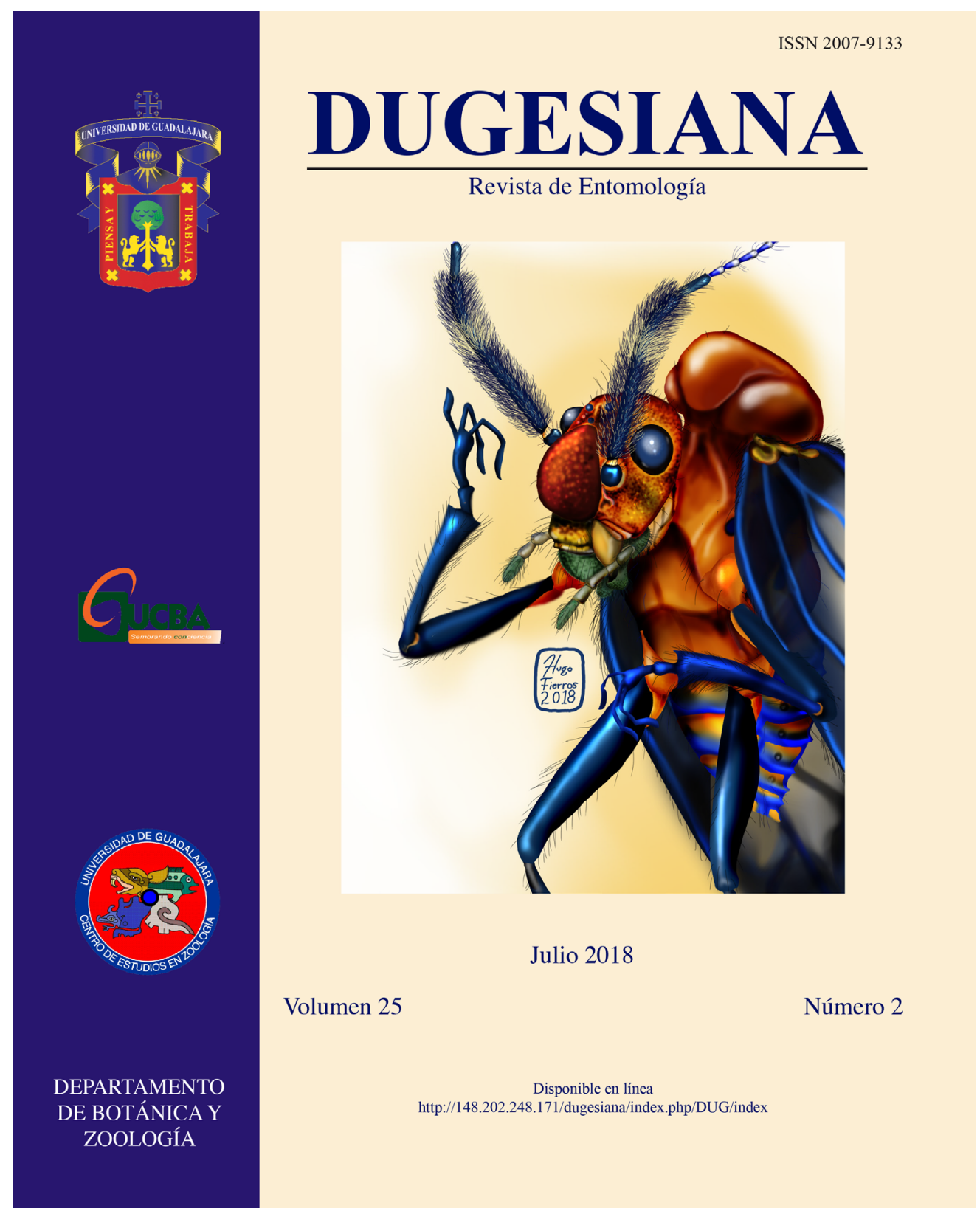

Dugesiana, Año 25, No. 2, julio 2018-diciembre 2018 (segundo semestre de 2018), es una publicación semestral, editada por la Universidad de Guadalajara, a través del Centro de Estudios en Zoología, por el Centro Universitario de Ciencias Biológicas y Agropecuarias. Camino Ramón Padilla Sánchez \# 2100, Nextipac, Zapopan, Jalisco, Tel. 37771150 ext. 33218, http://148.202.248.171/dugesiana/index.php/DUG/index, glenusmx@gmail.com. Editor responsable: José Luis Navarrete Heredia. Reserva de Derechos al Uso Exclusivo 04-2009-062310115100203, ISSN: 2007-9133, otorgados por el Instituto Nacional del Derecho de Autor. Responsable de la última actualización de este número: José Luis Navarrete Heredia, Editor y Ana Laura González-Hernández, Asistente Editorial. Fecha de la última modificación 25 de julio 2018, con un tiraje de un ejemplar.

Las opiniones expresadas por los autores no necesariamente reflejan la postura del editor de la publicación.

Queda estrictamente prohibida la reproducción total o parcial de los contenidos e imágenes de la publicación sin previa autorización de la Universidad de Guadalajara. 


\title{
Checklist of 'Psocoptera' (Psocodea) of Colombia and identification key to the families
}

\author{
Listado de especies de 'Psocoptera' (Psocodea) de Colombia y clave de identificación de las \\ familias
}

\author{
Alfonso Neri García Aldrete ${ }^{1 *}$, Nancy Soraya Carrejo ${ }^{2}$, Julián Mendivil ${ }^{2}$, Nadia Calderón², Oscar Saenz ${ }^{2}$, \\ Jeferson Panche², Cristian Román², Ranulfo González Obando² \\ 'Departamento de Zoología, Instituto de Biología, Universidad Nacional Autónoma de México, Apdo. Postal 70-153, CdMx, \\ MÉXICO. E-mail: anga@ib.unam.mx; ㅈupo de Investigaciones Entomológicas, Departamento de Biología, Facultad de \\ Ciencias Naturales y Exactas, Universidad del Valle, Santiago de Cali, COLOMBIA. E-mail: ranulfo.gonzalez@correounivalle. \\ edu.co,nancy.carrejo@correounivalle.edu.co \\ *Corresponding autor
}

\begin{abstract}
RESUMEN
Presentamos un listado de 720 especies de 'Psocoptera' registradas en Colombia, con base en la revisión de especímenes depositados en las colecciones del Instituto Alexander von Humboldt y el Museo Entomológico de la Universidad del Valle. Incluimos registros de ejemplares recientemente recolectados en diferentes Parques Naturales Nacionales y localidades adicionales en Colombia. Especies previamente registradas en la literatura también son incluidas en este listado. El número de psocópteros registrados hasta 2002 (79 especies) correspondía al 11\% de lo que es actualmente conocido con base en nuestro estudio. Enfatizamos que cerca del $60 \%$ de las especies registradas desde 2010 no habían sido descritas, 135 de las cuales fueron recientemente descritas por nuestro grupo de investigación. Incluimos una clave de identificación para las 28 familias de psócidos conocidas en Colombia. A pesar de que los psócidos han sido fuertemente marginados en la investigación taxonómica en Colombia, mostramos que la diversidad del grupo esta aún por ser descubierta en el pais. Estudios subsecuentes deberán ser enfocados a entender el patrón general de la distribución de psocópteros dentro y entre localidades, y enfatizamos la necesidad de conducir trabajo de campo extensivo.
\end{abstract}

Palabras clave: Diversidad, Lista, Neotrópico, Riqueza de especies.

\begin{abstract}
We present a list of 720 species of 'Psocoptera' recorded in Colombia, based on the examination of specimens deposited in the collections of the Alexander von Humboldt Institute and in the Entomological Museum of the Universidad del Valle. We include records of specimens recently collected in several National Natural Parks and other natural areas in Colombia. Species previously recorded in the literature are also listed here. The number of psocids recorded in Colombia until 2002 (79 species) corresponded to only $11.0 \%$ of what is currently known based on our study. We highlight that about $60 \%$ of the species recorded since 2010 were undescribed, 135 of which have been recently described by our research group. An identification key to the 28 psocid families known in Colombia is included. Even though psocids have been largely ignored from the taxonomic research in Colombia, we show that the group diversity is still to be uncovered in the country. Further studies must be conducted to understand the distribution of psocids among and within localities, and emphasize that additional extensive fieldwork is highly required.
\end{abstract}

Key words: Diversity, List, Neotropics, Species richness.

Lienhard and Smithers (2002) documented for Colombia 79 especies of 'Psocoptera' (41 genera and 19 families), most of which were collected by Professor Sturm, and identified and described by Badonnel (1986) almost 20 years before. Given the megadiverse nature of the country, we suspected that the psocid fauna was strongly underestimated. We therefore started a program in 2010 to sample and identify psocids. Initial fieldwork was conducted in the National Natural Park Gorgona Island, Cauca, and in natural areas of Valle del Cauca. These studies resulted in a list of 75 species for Gorgona Island (Sarria S., 2011; Sarria-S. et al., 2014a, b), and 85 species in four families of Epipsocetae (García
Aldrete et al.,, 2013 b). In both cases, a large number of undescribed species was detected, some of which have been described since (García Aldrete 2011, García Aldrete et al . 2011a, 2011b, 2012a, 2012b, 2013a, 2013b, 2013c, 2015; González et al. 2011a, 2011b, 2012, 2013, 2014, Calderón et al. 2014; Román-Palacios et al. 2014, Saenz et al. 2014, 2015). From the results of recent explorations, and from the examination of specimens collected in the frame of several projects, deposited in the collections of the Alexander von Humboldt Institute and at the Universidad del Valle, we elaborated the list of species occurring in Colombia. This checklist is presented below and includes 135 species 
recently described by some of us (García Aldrete 2012; García Aldrete et al. 2016; González et al. 2015a, 2015b, 2016, 2017a, b, c; Saenz et al. 2015; Mendivil-Nieto et al. 2017, Román-Palacios et al. 2015, 2016a, b; García Aldrete \& Román Palacios, 2015, Sandoval et al. 2016).

\section{MATERIAL AND METHODS}

In addition to the examination of specimens deposited in the collections indicated above, we collected extensively in the following parks and natural areas: National Natural Park (NNP hereafter) Gorgona Island (Cauca), NNP Cueva de Los Guácharos (Huila), NNP Los Katios (AntioquiaChocó), NNP La Paya (Putumayo), NNP La Macarena (Meta), NNP Los Farallones (Valle del Cauca), NNP Tamá (Norte de Santander), NNP Tayrona (Magdalena), OtúnQuimbaya Flora and Fauna Sanctuary (Risaralda), Natural Reserve El Encanto (Huila), Natural Reserve Pericos (Valle del Cauca), Natural Reserve Río Blanco (Caldas), Natural Reserve Chicaque-Tirolesa (Cundinamarca), Natural Reserve Planes de San Rafael (Risaralda), Natural Reserve Meremberg (Huila), Natural Reserve El Caduceo (Meta), Natural Reserve Río Negro (Risaralda), and in natural areas in Valle del Cauca (Buenaventura, Dagua, El Dovio, La Cumbre, Santiago de Cali, Tuluá), Caquetá (Belén de Los Andaquíes, San Vicente del Caguán), Putumayo (Puerto Asís, Puerto Leguizamo, Mocoa), Magdalena (San Javier) and Resguardos Indígenas Altamira (Caquetá), Tukunare (Putumayo), Kiwe (Caquetá) and La Esperanza (Caquetá).

Sampling was conducted by visual inspection on tree trunks and rock surfaces, beating vegetation, and indirectly with led light traps, placed in the canopy and operated at night for about 11-12 hours. Specimens were placed in vials with $80 \%$ ethanol. Some of the collected specimens were dissected, and their parts (head, wings, legs and genitalia) were mounted on slides in Canada Balsam following standard procedures.

\section{RESULTS}

The list below includes 720 species, about $60 \%$ of which are undescribed (426 especies), some of the families require a more detailed study, so the number of species might increase. The species marked with * correspond to those listed by Lienhard and Smithers (2002) or in additional literature; some of them were not found in our survey.

\section{Suborder Trogiomorpha \\ Family Lepidopsocidae}

Genus Echmepteryx Aaron, 1886

1. Echmepteryx falco (Badonnel, 1949). Cauca (NNP Gorgona Island), Valle del Cauca (Buenaventura).

2. Echmepteryx madagascariensis (Kolbe, 1885). Cauca (NNP Gorgona Island), Valle del Cauca (Buenaventura).

\section{Genus Lepidopsocus Enderlein, 1903}

3. Lepidopsocus pretiosus (Banks, 1942). Cauca (NNP Gorgona Island).
Genus Lepolepis Enderlein, 1906

4. Lepolepis columbiensis Badonnel, 1986*. Tolima (Icononzo).

Genus Proentomum Badonnel, 1949

5. Proentomum personatum Badonnel, 1949. Cauca (NNP Gorgona Island)

Genus Nepticulomima Enderlein, 1906

6. Nepticulomima hoesemanni (Enderlein, 1903). Cauca (NNP Gorgona Island).

Genus Soa Enderlein, 1904

7. Soa sp. 1. Valle del Cauca (Santiago de Cali).

Family Psoquillidae

Genus Psoquilla Hagen, 1865

8. Psoquilla marginepunctata Hagen, 1865. Chocó (NNP Los Katios).

Genus Rhyopsocus Hagen, 1876

9. Rhyopsocus bicornis Badonnel, 1986*. Magdalena (Capote).

\section{Famiy Psyllipsocidae \\ Genus Psocathropos Ribaga, 1899}

10. Psocathropos lachlani Ribaga, 1899*. Colombia.

Genus Psyllipsocus Selys- Longchamps, 1872

11. Psyllipsocus variabilis Badonnel, $1986^{*}$. Cundinamarca (subpáramo and páramo de Montserrate). Tolima (Icononzo).

\section{Suborder Troctomorpha \\ Family Amphientomidae \\ Genus Seopsocus Roesler, 1940}

12. Seopsocus sp. Cauca (NNP Gorgona Island).

\section{Genus Neoseopsis Badonnel, 1986}

13. Neoseopsis nuda Badonnel, 1986*. Cundinamarca (Páramo de Montserrate).

Genus Amphientomum Pictet, 1854

14. Amphientomum sp. Valle del Cauca (Cali), Risaralda (FFS Otún-Quimbaya).

\section{Family Compsocidae}

Genus Compsocus Banks, 1930

15. Compsocus elegans Banks, 1930. Cauca (NNP Gorgona Island).

Family Electrentomidae

Genus Epitroctes Mockford, 1967

16. Epitroctes sp. 1. Huila (NNP Cueva de Los Guácharos). Valle del Cauca (Santiago de Cali). 
17. Epitroctes sp. 2. Huila (NNP Cueva de Los Guácharos). Norte de Santander (NNP Tamá).

\section{Family Liposcelididae}

Genus Belaphotroctes Roesler, 1943

18. Belaphotroctes ghesquierei Badonnel, 1986*. Meta (Los Salados, ca. Villavicencio).

19. Belaphotroctes sp. Cauca (NNP Gorgona Island).

\section{Genus Belaphopsocus Badonnel, 1955}

20. Belaphopsocus badonneli New, 1971*. Tolima (Icononzo).

Genus Liposcelis Motschulsky, 1852

21. Liposcelis sp. Valle del Cauca (Dagua).

22. Liposcelis alticolis Badonnel, 1986*.

Cundinamarca (Páramo de Montserrate, Páramo El Tablazo, ca. Bogotá, Páramo de Sumapaz, Páramo de Chicasé). Nariño, Páramo de Cumbal.

23. Liposcelis bogotana Badonnel, 1986*. Cundinamarca (Bogotá).

24. Liposcelis bostrychophila Badonnel, 1931*. Meta. (Llanos Orientales, ca. Los Salados, Río Humea), Cundinamarca (Páramo de Montserrate).

25. Liposcelis dentata Badonnel, 1986*.

Cundinamarca (Bogotá).

26. Liposcelis entomophila Enderlein, 1907*. Meta (Llanos Orientales, ca. Los Salados, Río Humea). Tolima (Icononzo). Cundinamarca (Páramo de Montserrate).

27. Liposcelis fallax Badonnel, 1986*. Cundinamarca (Páramo de Sumapaz).

28. Liposcelis mimula Badonnel, 1986*. Boyacá (Cocuy).

29. Liposcelis ornata Mockford, 1978*. Cundinamarca (Subpáramo de Montserrate).

30. Liposcelis villosa Mockford, 1971*. Cundinamarca (Páramo de Montserrate).

Family Musapsocidae

Genus Musapsocus Mockford, 1967

31. Musapsocus sp. 1.Valle del Cauca (Dagua, Santiago de Cali).

32. Musapsocus sp. 2. Cauca (NNP Munchique).

Family Pachytroctidae

Genus Peritroctes Ribaga, 1911

33. Peritroctes sp. Valle del Cauca (Santiago de Cali).

Genus Pachytroctes Enderlein, 1905

34. Pachytroctes ixtapaensis García Aldrete, 1986. Valle del Cauca (Santiago de Cali, Campus Universidad del Valle).

Genus Tapinella Enderlein, 1908

35. Tapinella columbiana Badonnel, 1986*. Magdalena (Capote).

36. Tapinella sp. Cauca (NNP Gorgona Island). Valle del Cauca (Dagua, Santiago de Cali).

Genus Antilopsocus Gurney, 1965

37. Antilopsocus nadleri Gurney, 1965. Cauca (Caldono).

Family Troctopsocidae

Genus Troctopsocus Mockford, 1967

38. Troctopsocus sp. Norte de Santander (NNP Tamá).

Genus Troctopsocopsis Mockford, 1967

39. Troptopsocopsis sp. Risaralda (FFS Otún-Quimbaya).

Suborder Psocomorpha
Infraorder Epipsocetae
Family Cladiopsocidae

Genus Cladiopsocus Roesler, 1940

40. Cladiopsocus altamiraensis González, García Aldrete and Carrejo, 2018. Caquetá (San Vicente del Caguán).

41. Cladiopsocus amacayacuensis González, García Aldrete and Carrejo, 2016. Amazonas (NNP Amacayacu).

42. Cladiopsocus arboricolus Calderón, García Aldrete and González, 2014. Valle del Cauca (Dagua, Santiago de Cali, Tuluá).

43. Cladiopsocus belenensis González, García Aldrete and Carrejo, 2018. Caquetá (Belén de Los Andaquíes).

44. Cladiopsocus bidentatus Calderón, García Aldrete and González, 2014. Cauca (NNP Gorgona Island).

45. Cladiopsocus caguanensis González, García Aldrete and Carrejo, 2018. Caquetá (San Vicente del Caguán).

46. Cladiopsocus deliciasensis González, García Aldrete and Carrejo, 2016. Putumayo (Puerto Asís).

47. Cladiopsocus eertmoedi González, García Aldrete and Carrejo, 2016. Putumayo (Puerto Asís).

48. Cladiopsocus gregoriorum González, García Aldrete and Carrejo, 2016. Amazonas (NNP Amacayacu).

49. Cladiopsocus iscuande González, García Aldrete and Carrejo, 2018. Nariño (Iscuandé).

50. Cladiopsocus julianae González, García Aldrete and Carrejo, 2016. Putumayo (Mocoa). Caquetá (Belén de Los Andaquíes, San Vicente del Caguán).

51. Cladiopsocus katios González, García Aldrete and Carrejo, 2018. Chocó (NNP Los Katios).

52. Cladiopsocus kogui González, García Aldrete and Carrejo, 2018. Magdalena (Ciénaga, San Javier).

53. Cladiopsocus laesperanza González, García Aldrete and Carrejo, 2018. Caquetá (Belén de Los Andaquíes, San Vicente del Caguán).

54. Cladiopsocus mocoaensis González, García Aldrete and Carrejo, 2016. Putumayo (Mocoa). Caquetá (Belén de Los Andaquíes).

55. Cladiopsocus multimaculatus Eertmoed, 1986. Cauca (NNP Gorgona Island). Valle del Cauca (Buenaventura).

56. Cladiopsocus nebulosus Calderón, García Aldrete and González, 2014. Valle del Cauca (Dagua, El Dovio, Santiago de Cali, Tuluá). 
57. Cladiopsocus norsantanderinus González, García Aldrete and Carrejo, 2018. Norte de Santander (NNP Tamá).

58. Cladiopsocus pacificus Calderón, García Aldrete and González, 2014. Valle del Cauca (Buenaventura, Dagua).

59. Cladiopsocus panchei González, García Aldrete and Carrejo, 2016. Putumayo (Mocoa). Caquetá (Belén de Los Andaquíes, San Vicente del Caguán).

60. Cladiopsocus pancheorum González, García Aldrete and Carrejo, 2018. Putumayo (Puerto Asís).

61. Cladiopsocus ramulosus (Enderlein, 1903). Cundinamarca. Caquetá (Belén de Los Andaquíes, San Vicente del Caguán). Huila (Belén). Putumayo (Mocoa).

62. Cladiopsocus salasarriorum González, García Aldrete and Carrejo, 2018. Nariño (Iscuandé).

63. Cladiopsocus sixtoi González, García Aldrete and Carrejo, 2018. Caquetá (San Vicente del Caguán).

64. Cladiopsocus tikunus González, García Aldrete and Carrejo, 2016. Amazonas (Leticia, NNP Amacayacu). Cauca (Santa Leticia). Quindío (Filandia, Bosque de Bremen).

65. Cladiopsocus tinigua González, García Aldrete and Carrejo, 2018. Caquetá (Belén de Los Andaquíes, San Vicente del Caguán).

66. Cladiopsocus tukunare González, García Aldrete and Carrejo, 2018. Putumayo (Puerto Leguizamo).

\section{Family Dolabellopsocidae}

\section{Genus Dolabellopsocus Eertmoed, 1973}

67. Dolabellopsocus calima Calderón, García Aldrete and González, 2014. Valle del Cauca (Santiago de Cali, Tuluá).

68. Dolabellopsocus lobatus Mockford, 1991. Valle del Cauca (Santiago de Cali).

69. Dolabellopsocus roseusoides Calderón, García Aldrete and González, 2014. Valle del Cauca (Santiago de Cali).

70. Dolabellopsocus similis Mockford, 1991. Valle del Cauca (Santiago de Cali). Putumayo (Puerto Asís). Caquetá (San Vicente del Caguán).

71. Dolabellopsocus yundiguai Calderón, García Aldrete and González, 2014. Cauca (NNP Gorgona).

\section{Genus Isthmopsocus Eertmoed, 1973}

72. Isthmopsocus barbatusoides Calderón, García Aldrete and González, 2014. Cauca (NNP Gorgona). Valle del Cauca (Santiago de Cali).

73. Isthmopsocus foliatus Calderón, García Aldrete and González, 2014. Cauca (NNP Gorgona). Valle del Cauca (Santiago de Cali).

74. Isthmopsocus imperfectus* Badonnel, 1986. Magdalena (Capote).

75. Isthmopsocus sp.1. Caquetá (Belén de Los Andaquíes). Putumayo (Puerto Asís).

76. Isthmopsocus sp. 2. Putumayo (Puerto Asís).
Family Epipsocidae

Genus Anastomopsocus García Aldrete, Mendivil and González, 2015

77. Anastomopsocus vallecaucanus García Aldrete, Mendivil and González, 2015. Valle del Cauca (La Cumbre, Darién, Yumbo).

Genus Edmockfordia García Aldrete, 2009

78. Edmockfordia calderonae Mendivil, González and García Aldrete, 2015. Valle del Cauca (Buenaventura).

79. E. saenzi Mendivil, González and García Aldrete, 2015. Valle del Cauca (Buenaventura).

\section{Genus Epipsocus Hagen, 1866}

80. Epipsocus badonneli Mockford, 1991. Valle del Cauca (Santiago de Cali).

81. Epipsocus sp. 1. Valle del Cauca (Santiago de Cali).

82. Epipsocus sp. 2. Valle del Cauca (Santiago de Cali, Roldanillo).

83. Epipsocus sp. 3. Valle del Cauca (Santiago de Cali).

84. Epipsocus sp. 4. Valle del Cauca (Santiago de Cali, Darién), Risaralda (FFS Otún-Quimbaya).

85. Epipsocus sp. 5. Valle del Cauca (Buenaventura, Dagua).

86. Epipsocus sp. 6. Valle del Cauca (Buenaventura, Dagua).

87. Epipsocus sp. 7. Valle del Cauca (Buenaventura).

88. Epipsocus sp. 8. Valle del Cauca (Buenaventura).

89. Epipsocus sp. 9. Risaralda (FFS Otún-Quimbaya). Valle del Cauca (Santiago de Cali, Darién, El Cerrito, Roldanillo).

90. Epipsocus sp. 10. Valle del Cauca (Santiago de Cali).

91. Epipsocus sp. 11. Valle del Cauca (Santiago de Cali, Bolívar).

92. Epipsocus sp. 12. Valle del Cauca (Buenaventura, La Cumbre, Santiago de Cali).

93. Epipsocus sp. 13. Valle del Cauca (Santiago de Cali, Tuluá).

94. Epipsocus sp. 14. Valle del Cauca (Buenaventura, El Cerrito).

95. Epipsocus sp. 15. Valle del Cauca (Buenaventura, Roldanillo).

96. Epipsocus sp. 16. Valle del Cauca (Santiago de Cali).

97. Epipsocus sp. 17. Risaralda (FFS Otún-Quimbaya). Valle del Cauca (La Cumbre).

98. Epipsocus sp. 18. Valle del Cauca (Santiago de Cali).

99. Epipsocus sp. 19. Valle del Cauca (Buenaventura).

100. Epipsocus sp. 20. Valle del Cauca (Buenaventura, Dagua).

101. Epipsocus sp. 21. Amazonas (NNP Amacayacu). Huila (NR Meremberg).

102. Epipsocus sp. 22. Amazonas (NNP Amacayacu).

103. Epipsocus sp. 23. Putumayo (Puerto Asís).

104. Epipsocus sp. 24. Putumayo (Puerto Asís).

105. Epipsocus sp. 25. Putumayo (Puerto Asís).

106. Epipsocus sp. 26. Putumayo (Puerto Asís). 
107. Epipsocus sp. 27. Putumayo (Puerto Asís).

108. Epipsocus sp. 28. Huila (La Plata). Putumayo (Mocoa, Puerto Asis, Puerto Leguizamo).

109. Epipsocus sp. 29. Putumayo (Puerto Asís).

110. Epipsocus sp. 30. Putumayo (Mocoa, Puerto Leguizamo).

111. Epipsocus sp. 31. Putumayo (Puerto Asís).

112. Epipsocus sp. 32. Huila (NR Meremberg).

113. Epipsocus sp. 33. Vaupés (Igapó, Mosiro-Itajura).

114. Epipsocus sp. 34. Magdalena (NNP Tayrona).

115. Epipsocus sp. 35. Meta (NNP Serranía de La Macarena, Tinigua).

Genus Goja Navás, 1927

116. Goja bogotana (Roesler, 1940)*. Cundinamarca (Bogotá).

117. Goja sp. 1. Valle del Cauca (Darién, La Cumbre, Roldanillo, Santiago de Cali, Yotoco, Yumbo).

118. Goja sp. 2. Valle del Cauca (Santiago de Cali).

119. Goja sp. 3. Valle del Cauca (Jamundí, Santiago de Cali).

120. Goja sp. 4. Valle del Cauca (Santiago de Cali).

121. Goja sp. 5. Valle del Cauca (Santiago de Cali).

122. Goja sp. 6. Risaralda (FFS Otún-Quimbaya). Valle del Cauca (Santiago de Cali).

123. Goja sp. 7. Caldas (Manizales). Risaralda (FFS Otún-Quimbaya). Valle del Cauca (Sevilla).

124. Goja sp. 8. Risaralda (FFS Otún-Quimbaya). Valle del Cauca (Sevilla).

125. Goja sp. 9. Risaralda (FFS Otún-Quimbaya).

126. Goja sp. 10. Caldas (Manizales).

127. Goja sp. 11. Cauca (NNP Puracé).

128. Goja sp. 12. Huila (NR Meremberg).

129. Goja sp. 13. Norte de Santander (NNP Tamá).

130. Goja sp. 14. Norte de Santander (NNP Tamá).

Genus Gojaoides García Aldrete, 2012

131. Gojaoides aviceps (Badonnel, 1986). Huila (NNP Cueva de Los Guácharos).

132. Gojaoides sp. 1. Risaralda (FFS Otún-Quimbaya). Valle del Cauca (La Cumbre, Yumbo).

133. Gojaoides sp. 2. Boyacá (FFS Iguaqué). Valle del Cauca (Buenaventura, Dagua).

134. Gojaoides sp. 3. Risaralda (FFS Otún-Quimbaya). Valle del Cauca (La Cumbre, Yumbo).

135. Gojaoides sp. 4. Risaralda (SFF Otún-Quimbaya), Valle del Cauca (La Cumbre, Yumbo).

136. Gojaoides sp. 5. Valle del Cauca (La Cumbre, Santiago de Cali, Yumbo).

137. Gojaoides sp. 6. Valle del Cauca (Roldanillo).

138. Gojaoides sp. 7. Valle del Cauca (El Cerrito).

139. Gojaoides sp. 8. Valle del Cauca (La Cumbre).

140. Gojaoides sp. 9. Valle del Cauca (Jamundí, Roldanillo, Santiago de Cali).

141. Gojaoides sp. 10. Valle del Cauca (Sevilla).

142. Gojaoides sp. 11. Valle del Cauca (Sevilla).
143. Gojaoides sp. 12. Risaralda (FFS Otún-Quimbaya).

144. Gojaoides sp. 13. Risaralda (FFS Otún-Quimbaya).

145. Gojaoides sp. 14. Caldas (Manizales). Risaralda (FFS Otún-Quimbaya).

146. Gojaoides sp. 15. Cauca (NNP Gorgona Island).

147. Gojaoides sp. 16. Risaralda (Planes de San Rafael Sanctuary).

148. Gojaoides sp. 17. Risaralda (Planes de San Rafael Sanctuary).

149. Gojaoides sp. 18. Risaralda (Planes de San Rafael Sanctuary).

150. Gojaoides sp. 19. Caldas (Pensilvania).

151. Gojaoides sp. 20. Huila (NR Meremberg).

152. Gojaoides sp. 21 Huila (NR Meremberg).

153. Gojaoides sp. 22. Huila (NR Meremberg).

154. Gojaoides sp. 23. Huila (NNP Cueva de Los Guácharos).

155. Gojaoides sp. 24. Huila (NNP Cueva de Los Guácharos).

156. Gojaoides sp. 25. Meta (NNP Serranía de La Macarena).

157. Gojaoides sp. 26. Boyacá (FFS Iguaqué).

158. Gojaoides sp. 27. Cundinamarca (NNP Chingaza).

159. Gojaoides sp. 28. Cundinamarca (NNP Chingaza).

160. Gojaoides sp. 29. Huila (NNP Cueva de Los Guácharos).

161. Gojaoides sp. 30. Boyacá (FFS Iguaqué).

162. Gojaoides sp. 31. Magdalena (NNP Sierra Nevada de Santa Marta).

163. Gojaoides sp. 32. Magdalena (NNP Sierra Nevada de Santa Marta).

164. Gojaoides sp. 33. Magdalena (NNP Sierra Nevada de Santa Marta).

165. Gojaoides sp. 34. Putumayo (Puerto Asís).

166. Gojaoides sp. 35. Putumayo (Puerto Asís).

167. Gojaoides sp. 36. Putumayo (Mocoa).

168. Gojaoides sp. 37. Putumayo (Puerto Asís).

169. Gojaoides sp. 38. Putumayo (Puerto Asís).

170. Gojaoides sp. 39. Norte de Santander (NNP Tamá).

171. Gojaoides sp. 40. Norte de Santander (NNP Tamá).

172. Gojaoides sp. 41. Norte de Santander (NNP Tamá).

173. Gojaoides sp. 42. Norte de Santander (NNP Tamá).

174. Gojaoides sp. 43. Norte de Santander (NNN Tamá).

175. Gojaoides sp. 44. Norte de Santander (NNP Tamá).

176. Gojaoides sp. 45. Norte de Santander (NNP Tamá).

177. Gojaoides sp. 46. Norte de Santander (NNP Tamá).

178. Gojaoides sp. 47. Norte de Santander (NNP Tamá).

179. Gojaoides sp. 48. Risaralda (Santa Rosa de Cabal).

180. Gojaoides sp. 49. Putumayo (Puerto Asís).

Genus Gonzobandia García Aldrete, 2012

181. Gonzobandia nancyae García Aldrete, 2012. Valle del Cauca (La Cumbre, Santiago de Cali, Yumbo).

Genus Mesepipsocus Badonnel, 1969

182. Mesepipsocus brasiliensis (New, 1980). Amazonas 
(NNP Amacayacu).

183. Mesepipsocus brevistigma (New, 1972). Valle del Cauca (Ansermá Nuevo).

184. Mesepipsocus campanulatus (Thornton \& Woo, 1973). Valle del Cauca (Buenaventura, Dagua).

185. Mesepipsocus fuscatus (New, 1972). Putumayo (Puerto Asís).

186. Mesepipsocus proctus (New and Thornton, 1988). Putumayo (Puerto Asís).

187. Mesepipsocus umbratus (New and Thornton, 1988). Valle del Cauca (Yumbo, Dagua, Sevilla).

188. Mesepipsocus sp. 1. Valle del Cauca (Santiago de Cali).

189. Mesepipsocus sp. 2. Valle del Cauca (La Cumbre).

190. Mesepipsocus sp. 3. Valle del Cauca (Buenaventura, Santiago de Cali).

191. Mesepipsocus sp. 4. Valle del Cauca (Dagua).

192. Mesepipsocus sp. 5. Amazonas (NNP Amacayacu).

193. Mesepipsocus sp. 6. Amazonas (NNP Amacayacu).

194. Mesepipsocus sp. 7. Huila (NNP Cueva de Los Guácharos).

195. Mesepipsocus sp. 8. Huila (NNP Cueva de Los Guácharos).

196. Mesepipsocus sp. 9. Putumayo (Mocoa).

\section{Genus Neurostigma Enderlein, 1900}

197. Neurostigma furcivenula Badonnel, 1986*. Magdalena (Capote).

198. Neurostigma xanthopterum New, 1980. Vaupés (Igapó, Mosiro-Itajura).

199. Neurostigma sp. 1. Valle del Cauca (Santiago de Cali).

200. Neurostigma sp. 2. Boyacá (NNP Iguaqué). Valle del Cauca (Santiago de Cali).

201. Neurostigma sp. 3. Magdalena (NNP Tayrona). Valle del Cauca (Dagua).

202. Neurostigma sp. 4. Putumayo (Puerto Asís).

203. Neurostigma sp. 5. Huila (NR Meremberg).

204. Neurostigma sp. 6. Amazonas (NNP Amacayacu). Vaupés (Igapó, Mosiro-Itajura).

\section{Genus Odontopsocus Badonnel, 1987}

205. Odontopsocus compactus Lienhard, 2002*. Magdalena (Sierra Nevada de Santa Marta).

\section{Genus Parepipsocus Badonnel, 1986}

206. Parepipsocus obscurus Badonnel, Cundinamarca (Páramo de Montserrate).

\section{Genus Periepipsocus García Aldrete, Mendivil and González, 2015}

207. Periepipsocus caliensis García Aldrete, Mendivil and González, 2015. Valle del Cauca (Santiago de Cali).
Genus Terryerwinia García Aldrete, 2005

208. Terryerwinia acutiphallica García Aldrete, 2005. Putumayo (Puerto Asís).

\section{New genus 1}

209. New genus 1. sp. 1. Valle del Cauca (Dagua).

210. New genus 1. sp. 2. Valle del Cauca (Buenaventura).

211. New genus 1. sp. 3. Putumayo (Puerto Asís). Valle del Cauca (Dagua).

212. New genus 1.sp. 4. Amazonas (NNP Amacayacu). Putumayo (Puerto Asís, Puerto Leguizamo).

213. New genus 1. sp. 5. Amazonas (NNP Amacayacu). Putumayo (Mocoa, Puerto Asis).

214. New genus1. sp. 6. Amazonas (NNP Amacayacu). Caquetá (ca. NNP Chiribiquete). Meta (Serranía de La Macarena).

215. New genus 1. sp. 7. Meta (Río Oloroso). Putumayo (Puerto Leguizamo).

216. New genus 1. sp. 8. Meta (Serranía de La Macarena).

217. New genus 1. sp. 9. Putumayo (NNP La Paya). Vaupés (Igapó, Mosiro-Itajura).

218. New genus 1. sp. 10. Putumayo (Puerto Asis). Vaupés (Igapó, Mosiro-Itajura).

219. New genus 1. sp. 11. Putumayo (Puerto Asís).

220. New genus 1. sp. 12. Putumayo (Puerto Leguizamo).

221. New genus 1. sp. 13. Putumayo (Puerto Asís).

222. New genus 1. sp. 14. Putumayo (Puerto Asís).

223. New genus 1. sp. 15. Putumayo (Mocoa).

224. New genus 1. sp. 16. Putumayo (Puerto Asís).

\section{Family Ptiloneuridae}

New Genus

225. New genus sp. 1. Cundinamarca (NR Chicaque).

226. New genus sp. 2. Huila (NR Meremberg, Cascada La Candelaria).

227. New genus sp. 3. Valle del Cauca (Santiago de Cali). Risaralda ( Planes de San Rafael Sanctuary).

228. New genus sp. 4. Huila (NR Meremberg).

229. New genus sp. 5. Norte de Santander (NNP Tamá).

\section{Genus Euplocania Enderlein, 1910}

230. Euplocania badonneli New and Thornton, 1988. Amazonas (NNP Amacayacu). Caquetá (San Vicente del Caguán, Jericó-Consayá). Putumayo (Puerto Asís).

231. Euplocania bonaverensis González, García Aldrete and Carrejo, 2015. Valle del Cauca (Buenaventura).

232. Euplocania caliensis González, García Aldrete and Carrejo, 2015. Valle del Cauca (Santiago de Cali, Ansermá Nuevo). Antioquia (Maceo).

233. Euplocania caquetaensis González, García Aldrete and Carrejo, 2017. Caquetá (Belén de Los Andaquíes, San Vicente del Caguán).

234. Euplocania daguaensis González, García Aldrete and Carrejo, 2015. Valle del Cauca (Dagua). 
235. Euplocania danubiana González, García Aldrete and Carrejo, 2015. Valle del Cauca (Buenaventura, Dagua).

236. Euplocania gaitana González, García Aldrete and Carrejo, 2017. Caquetá (Belén de Los Andaquíes).

237. Euplocania guentherbuchi González, García Aldrete and Carrejo, 2015. Huila (NR Meremberg).

238. Euplocania laelsa González, García Aldrete and Carrejo, 2017. Valle del Cauca (Dagua).

239. Euplocania macarenaensis González, García Aldrete and Carrejo, 2015. Meta (NNP Serranía de La Macarena).

240. Euplocania nasa González, García Aldrete and Carrejo, 2017. Huila (NNP Cueva de Los Guácharos).

241. Euplocania reyesi García Aldrete, González and Carrejo, 2013. Magdalena (NNP Tayrona).

242. Euplocania vallecaucana González, García Aldrete and Carrejo, 2015. Valle del Cauca (Dagua).

243. Euplocania yalcona. González, García Aldrete and Carrejo, 2017. Huila (Palestina).

244. Euplocania sp. 1. Putumayo (Mocoa).

245. Euplocania sp. 2. Risaralda (NR Río Negro).

246. Euplocania sp. 3. Chocó (NNP Los Katios).

247. Euplocania sp. 4. Caquetá (Belén de Los Andaquíes).

248. Euplocania sp. 5. Caquetá (San Vicente del Caguán).

249. Euplocania sp. 6. Caquetá (San Vicente del Caguán).

250. Euplocania sp. 7. Caquetá (San Vicente del Caguán).

251. Euplocania sp. 8. Caquetá (San Vicente del Caguán).

252. Euplocania sp. 9. Putumayo (Puerto Asís).

253. Euplocania sp. 10. Caquetá (San Vicente del Caguán). Putumayo (Mocoa).

254. Euplocania sp. 11. Chocó (NNP Los Katios). Valle del Cauca (Buenaventura).

255. Euplocania sp. 12. Putumayo (Puerto Asis).

256. Euplocania sp. 13. Vaupés (Caparú, Mosiro-Itajura).

257. Euplocania sp. 14. Amazonas (NNP Amacayacu).

258. Euplocania sp. 15. Amazonas (NNP Amacayacu). Putumayo (Puerto Leguizamo).

259. Euplocania sp. 16. Putumayo (Puerto Leguizamo).

260. Euplocania sp. 17. Caquetá (San Vicente del Caguán).

261. Euplocania sp. 18. Meta (NNP Serranía de La Macarena).

\section{Genus Loneura Navás, 1927}

262. Loneura andina García Aldrete, Mendivil and González, 2012. Valle del Cauca (Santiago de Cali). Risaralda (FFS Otún-Quimbaya).

263. Loneura colombiana García Aldrete, González and Carrejo, 2011. Magdalena (NNP Tayrona). Meta (Villavicencio).

264. Loneura dapaensis Mendivil, García Aldrete and González, 2017. Valle del Cauca (La Cumbre). Risaralda (Planes de San Rafael Sanctuary, NR Pueblo Rico).

265. Loneura deibyi Mendivil, García Aldrete and González, 2017. Valle del Cauca (Dagua, Santiago de
Cali, El Dovio).

266. Loneura eberhardi Mendivil, García Aldrete and González, 2017. Valle del Cauca (Santiago de Cali, Dagua). Risaralda (NR Río Negro).

267. Loneura farallonensis Mendivil, García Aldrete and González, 2017. Valle del Cauca (Santiago de Cali).

268. Loneura gorgonaensis García Aldrete, González and Sarria, 2011. Cauca. (NNP Gorgona Island). Valle del Cauca (Buenaventura, Dagua).

269. Loneura insularis García Aldrete, González and Sarria, 2011. Cauca (NNP Gorgona Island).

270. Loneura mirandaensis García Aldrete, 2007. Valle del Cauca (Santiago de Cali).

271. Loneura monticola García Aldrete, González and Sarria, 2011. Cauca (NNP Gorgona Island).

272. Loneura montserrate Mendivil, García Aldrete and González, 2017. Valle del Cauca (Santiago de Cali). Risaralda (Planes de San Rafael Sanctuary, NR Río Negro).

273. Loneura quimbaya Mendivil, García Aldrete and González, 2017. Risaralda (FFS Otún- Quimbaya).

274. Loneura tuluaensis García Aldrete, Mendivil and González, 2012. Valle del Cauca (Tuluá, El Dovio).

275. Loneura univalle Mendivil, García Aldrete and González, 2017. Valle del Cauca (Santiago de Cali). Caquetá (San Vicente del Caguán).

276. Loneura sp. 1. Magdalena (NNP Tayrona).

277. Loneura sp. 2. Valle del Cauca (Dagua). Risaralda (NR Río Negro).

278. Loneura sp. 3. Magdalena (NNP Tayrona).

279. Loneura sp. 4. Putumayo (Puerto Asís).

280. Loneura sp. 5. Putumayo (Puerto Asís).

281. Loneura sp. 6. Magdalena (NNP Tayrona, Ciénaga, San Javier).

282. Loneura sp. 7. Magdalena (Ciénaga, San Javier).

283. Loneura sp. 8. Huila (NNP Cueva de Los Guácharos, NR Meremberg).

284. Loneura sp. 9. Huila (Palestina, La Mensura).

285. Loneura sp. 10. Risaralda (FFS Otún-Quimbaya).

286. Loneura sp. 11. Chocó (NNP Los Katios).

287. Loneura sp. 12. Caquetá (Belén de Los Andaquíes).

288. Loneura sp. 13. Caquetá (Belén de Los Andaquíes).

289. Loneura sp. 14. Caquetá (Belén de Los Andaquíes).

290. Loneura sp. 15. Caquetá (Belén de Los Andaquíes).

291. Loneura sp. 16. Caquetá (Belén de Los Andaquíes).

292. Loneura sp. 17. Caquetá (San Vicente del Caguán).

293. Loneura sp. 18. Caquetá (San Vicente del Caguán).

294. Loneura sp. 19. Putumayo (Puerto Asís).

295. Loneura sp. 20. Caquetá (San Vicente del Caguán).

296. Loneura sp. 21. Caquetá (San Vicente del Caguán).

297. Loneura sp. 22. Caquetá (San Vicente del Caguán).

298. Loneura sp. 23. Caquetá (San Vicente del Caguán).

299. Loneura sp. 24. Caquetá (San Vicente del Caguán).

\section{Genus Loneuroides García Aldrete, 2006}

300. Loneuroides bretanaensis García Aldrete, González 
and Carrejo, 2016. Risaralda (FFS Otún-Quimbaya). Valle del Cauca (Sevilla).

301. Loneuroides colombianus García Aldrete, González and Carrejo, 2016. Cundinamarca (NR ChicaqueTirolesa). Valle del Cauca (Sevilla).

302. Loneuroides ledesmai García Aldrete, González and Carrejo, 2016. Risaralda (Planes de San Rafael Sanctuary, FFS Otún-Quimbaya).

303. Loneuroides rionegroensis García Aldrete, González and Carrejo, 2016. Risaralda (NR Río Negro).

304. Loneuroides santanderinus García Aldrete, González and Carrejo, 2016. Norte de Santander (NNP Tamá).

305. Loneuroides tamaensis García Aldrete, González and Carrejo, 2016. Norte de Santander (NNP Tamá).

306. Loneuroides venezolanus García Aldrete, 2006. Valle del Cauca (La Cumbre).

307. Loneuroides sp. 1. Huila (NR Meremberg).

Genus Perucania New and Thornton, 1988

308. Perucania longiareola New and Thornton, 1988. Caquetá (Macarena, Resguardo Indigena Kiwe).

\section{Genus Ptiloneura Enderlein, 1900}

309. Ptiloneura bidorsalis Enderlein, 1900. Huila (NNP Cueva de Los Guácharos).

\section{Genus Triplocania (Roesler, 1940)}

310. Triplocania amacayacuensis González, Carrejo and García Aldrete, 2017. Amazonas (NNP Amacayacu).

311. Triplocania anchicayaensis González, Carrejo and García Aldrete, 2017. Valle del Cauca (Buenaventura).

312. Triplocania andaqui González, Carrejo and García Aldrete, 2017. Huila (NNP Cueva de Los Guácharos).

313. Triplocania arhuaca González, Carrejo and García Aldrete, 2017. Magdalena (NNP Tayrona).

314. Triplocania asisensis González, Carrejo and García Aldrete, 2017. Putumayo (Puerto Asís).

315. Triplocania awa González, Carrejo and García Aldrete, 2017. Putumayo (Mocoa). Caquetá (Belén de Los Anadaquíes).

316. Triplocania bicornuta González, Carrejo and García Aldrete, 2017. Valle del Cauca (Buenaventura, Dagua).

317. Triplocania bubuae González, Carrejo and García Aldrete, 2017. Valle del Cauca (Buenaventura).

318. Triplocania calima González, Carrejo and García Aldrete, 2017. Valle del Cauca (Santiago de Cali).

319. Triplocania camentsa González, Carrejo and García Aldrete, 2017. Putumayo (Mocoa). Caquetá (Belén de Los Anadaquíes).

320. Triplocania cantatis González, Carrejo and García Aldrete, 2017. Huila (Palestina: NR El Encanto).

321. Triplocania caribe González, Carrejo and García Aldrete, 2017. Norte de Santander (NNP Tamá).

322. Triplocania chocoensis González, Carrejo and García Aldrete, 2017. Valle del Cauca (Buenaventura).
323. Triplocania dimitrii González, Carrejo and García Aldrete, 2017. Cundinamarca (Soacha: NR Chicaque).

324. Triplocania embera González, Carrejo and García Aldrete, 2017. Valle del Cauca (Buenaventura).

325. Triplocania erwini Silva-Neto, Rafael and García Aldrete, 2015. Amazonas (NNP Amacayacu). Putumayo (Puerto Asís).

326. Triplocania felidiaensis González, Carrejo and García Aldrete, 2017. Valle del Cauca (Jamundí, Santiago de Cali).

327. Triplocania furcata New, 1972. Amazonas (NNP Amacayacu). Putumayo (Puerto Leguizamo).

328. Triplocania furcatoides González, Carrejo and García Aldrete, 2017. Amazonas (NNP Amacayacu).

329. Triplocania garciamarquezi González, Carrejo and García Aldrete, 2017. Valle del Cauca (Roldanillo).

330. Triplocania guane González, Carrejo and García Aldrete, 2017. Norte de Santander (NNP Tamá).

331. Triplocania huilaensis González, Carrejo and García Aldrete, 2017. Huila (NR Meremberg).

332. Triplocania huitota González, Carrejo and García Aldrete, 2017. Putumayo (Puerto Asís). Caquetá (San Vicente del Caguán).

333. Triplocania humboldtiana González, Carrejo and García Aldrete, 2017. Norte de Santander (NNP Tamá).

334. Triplocania inga González, Carrejo and García Aldrete, 2017. Putumayo (Mocoa).

335. Triplocania kichwa González, Carrejo and García Aldrete, 2017. Putumayo (Puerto Asís).

336. Triplocania korebaju González, Carrejo and García Aldrete, 2017. Putumayo (Puerto Asís).

337. Triplocania lamasi Silva-Neto, Rafael and García Aldrete, 2014. Putumayo (Puerto Asís, Puerto Leguizamo, NNP La Paya).

338. Triplocania lamasoides Silva-Neto, Rafael and García Aldrete, 2015. Caquetá (San Vicente del Caguán, Resguardo Indigena Yaguara).

339. Triplocania lamensuraensis González, Carrejo and García Aldrete, 2017. Huila (Palestina, La Mensura).

340. Triplocania lapayaensis González, Carrejo and García Aldrete, 2017. Putumayo (Puerto Leguizamo, NNP La Paya).

341. Triplocania leguizamoensis González, Carrejo and García Aldrete, 2017. Putumayo (Puerto Leguizamo).

342. Triplocania litophila González, Carrejo and García Aldrete, 2017. Valle del Cauca (La Cumbre).

343. Triplocania maricarmenae González, Carrejo and García Aldrete, 2017. Caldas (Manizales).

344. Triplocania matildae González, Carrejo and García Aldrete, 2017. Huila (NR Meremberg).

345. Triplocania mocoaensis González, Carrejo and García Aldrete, 2017. Putumayo (Mocoa).

346. Triplocania motilona González, Carrejo and García Aldrete, 2017. Norte de Santander (NNP Tamá).

347. Triplocania otunquimbayaensis González, Carrejo and García Aldrete, 2017. Risaralda (FFS Otún- 
Quimbaya).

348. Triplocania panchei González, Carrejo and García Aldrete, 2017. Putumayo (Mocoa). Caquetá (Belén de Los Andaquíes).

349. Triplocania pericosensis González, Carrejo and García Aldrete, 2017. Valle del Cauca (Buenaventura).

350. Triplocania robustoides González, Carrejo and García Aldrete, 2017. Putumayo (Puerto Asís).

351. Triplocania rugosa González, Carrejo and García Aldrete, 2017. Valle del Cauca (Buenaventura).

352. Triplocania sarmaca González, Carrejo and García Aldrete, 2017. Putumayo (NNP La Paya).

353. Triplocania sarriae González, Carrejo and García Aldrete, 2017. Valle del Cauca (Buenaventura, Dagua).

354. Triplocania sevillaensis González, Carrejo and García Aldrete, 2017. Valle del Cauca (Sevilla).

355. Triplocania yanacona González, Carrejo and García Aldrete, 2017. Risaralda (FFS Otún-Quimbaya, (NR Río Negro). Valle del Cauca (La Cumbre, Santiago de Cali). Caquetá (San Vicente del Caguán).

356. Triplocania sp. 1. Cauca (NNP Gorgona Island).

357. Triplocania sp. 2. Chocó (NNP Los Katios).

358. Triplocania sp. 3. Caquetá (Belén de Los Andaquíes).

359. Triplocania sp. 4. Caquetá (Belén de Los Andaquíes).

360. Triplocania sp. 5. Caquetá (Belén de Los Andaquíes).

361. Triplocania sp. 6. Caquetá (San Vicente del Caguán).

362. Triplocania sp. 7. Caquetá (San Vicente del Caguán).

363. Triplocania sp. 8. Caquetá (San Vicente del Caguán).

364. Triplocania sp. 9. Caquetá (San Vicente del Caguán).

365. Triplocania sp. 10. Caquetá (San Vicente del Caguán).

\section{Infraorder Caeciliusetae} Family Amphipsocidae

Genus Polypsocus Hagen, 1866

366. Polypsocus sp. 1. Norte de Santander (NNP Tamá).

367. Polypsocus sp. 2. Valle del Cauca (Dagua, Santiago de Cali).

368. Polypsocus sp 3. Valle del Cauca (Santiago de Cali).

\section{Family Asiopsocidae}

\section{Genus Notiopsocus Banks, 1913}

369. Notiopsocus sp. 1. Cauca (NNP Gorgona).

370. Notiopsocus sp. 2. Chocó (NNP Los Katios)

\section{Family Caeciliusidae}

Genus Coryphaca Enderlein, 1910

371. Coryphaca sp. 1. Valle del Cauca (Santiago de Cali). Huila (NNP Cueva de Los Guácharos).

372. Coryphaca sp. 2. Huila (NNP Cueva de Los Guácharos).

Genus Valenzuela Navás, 1924

373. Valenzuela andeanus (New and Thornton, 1975)*. Cundinamarca (Bogotá).

374. Valenzuela columbianus (Badonnel, 1986)*.
Magdalena (Capote).

375. Valenzuela paramonus (Badonnel, 1986)*. Cundinamarca (Páramo de Montserrate).

376. Valenzuela thiemei (Enderlein, 1903)*. Cordillera, Tierra fría.

377. Valenzuela sp. 1. Cauca (NNP Gorgona Island).

378. Valenzuela sp. 2. Cauca (NNP Gorgona Island).

379. Valenzuela sp. 3. Cauca (NNP Gorgona Island).

380. Valenzuela sp. 4. Cauca (NNP Gorgona Island).

381. Valenzuela sp. 5. Cauca (NNP Gorgona Island).

382. Valenzuela sp. 6. Cauca (NNP Gorgona Island).

\section{Genus Xanthocaecilius Mockford, 1989}

383. Xanthocaecilius montserratensis (Badonnel, 1986)*. Cundinamarca (Páramo de Montserrate).

Family Dasydemellidae

Genus Dasydemella Enderlein, 1909

384. Dasydemella montana Badonnel, 1986*. Cundinamarca, Páramo de Montserrate.

385. Dasydemella obrienorum García Aldrete, 1978. Valle del Cauca (Santiago de Cali).

386. Dasydemella sp. 1. Risaralda (FFS Otún-Quimbaya)

Genus Teliapsocus Chapman, 1930

387. Teliapsocus distinctus Badonnel, 1986*. Cundinamarca (Páramo de Palacio-Chisacá).

\section{Family Stenopsocidae}

\section{Genus Graphopsocus Kolbe, 1980}

388. Graphopsocus cruciatus (Linnaeus, 1768). Cauca (NNP Gorgona Island). Valle del Cauca (Dagua). Caquetá (San Vicente del Caguán).

\section{Infraorder Homilopsocidea Family Archipsocidae Genus Archipsocus Hagen, 1882}

389. Archipsocus sp. Valle del Cauca (Santiago de Cali).

390. Archipsocus badonneli New, 1973*. Meta (Los Salados, ca. Villavicencio). Magdalena (Capote).

391. Archipsocus castrii Badonnel, 1978*. Magdalena (Capote).

392. Archipsocus cervinus New, 1973*. Cundinamarca (Bogotá)

393. Archipsocus modestus New, 1973*. Magdalena (Capote).

Genus Pararchipsocus Badonnel, Mockford and García Aldrete, 1984

394. Pararchipsocus sp. Cauca (NNP Gorgona).

Family Ectopsocidae

Genus Ectopsocus McLachlan, 1899

395. Ectopsocus andinus Saenz, González and García Aldrete, 2014. Valle del Cauca (Santiago de Cali, Tuluá).

396. Ectopsocus briggsi McLachlan, 1899. Valle del 
Cauca (El Cerrito, La Cumbre).

397. Ectopsocus californicus Banks, 1903. Cauca. (NNP Gorgona Island). Valle del Cauca (La Cumbre).

398. Ectopsocus columbianus Badonnel, 1986. Meta (Los Salados, ca. Villavicencio). Valle del Cauca (Santiago de Cali).

399. Ectopsocus gorgonaensis Saenz, González and García Aldrete, 2014. Cauca (NNP Gorgona Island).

400. Ectopsocus maindroni Badonnel, 1935. Cauca (NNP Gorgona Island). Valle del Cauca (Santiago de Cali).

401. Ectopsocus meridionalis Ribaga, 1904. Cauca (NNP Gorgona Island). Boyacá, (Lago de Tota). Risaralda (FFS Otún-Quimbaya). Valle del Cauca (La Cumbre, El Cerrito, Santiago de Cali).

402. Ectopsocus pilosus Badonnel, 1967. Valle del Cauca (Dagua, Santiago de Cali).

403. Ectopsocus richardsi Pearman, 1929. Valle del Cauca (Santiago de Cali, Buenaventura).

404. Ectopsocus titschacki Jentsch, 1939. Cauca (NNP Gorgona Island).

405. Ectopsocus thorntoni García Aldrete, 1991. Cauca (NNP Gorgona Island). Chocó (NNP Los Katios).

406. Ectopsocus valvilobatus Saenz, González and García Aldrete, 2014. Cauca (NNP Gorgona Island).

407. Ectopsocus vilhenai Badonnel, 1955. Valle del Cauca (El Cerrito).

408. Ectopsocus vilhenaioides Saenz, González and García Aldrete, 2014. Valle del Cauca (Buenaventura, El Cerrito, Santiago de Cali).

409. Ectopsocus sp. 1. Risaralda (FFS Otún-Quimbaya)

\section{Genus Ectopsocopsis Badonnel, 1955}

410. Ectopsocopsis cryptomeriae (Enderlein). Valle del Cauca (Santiago de Cali).

\section{Family Lachesillidae Subfamily Lachesillinae \\ Genus Lachesilla Westwood, 1840 \\ Pedicularia species group}

411. Lachesilla aethiopica (Enderlein, 1902). Valle del Cauca (Santiago de Cali, Roldanillo).

412. Lachesilla aldretei Badonnel, 1986*. Meta (Llanos Orientales, ca. Los Salados and Río Humea). Tolima (Icononzo). Cundinamarca (Páramo de Montserrate).

413. Lachesilla amacayacuensis García Aldrete, 2010. Amazonas (NNP Amacayacu).

414. Lachesilla asymmetriproctus García Aldrete, 2008. Putumayo (Puerto Asís).

415. Lachesilla cesarcardonai García Aldrete, González and Saldaña, 2014. Valle del Cauca (Santiago de Cali).

416. Lachesilla nuptialis Badonnel and García Aldrete, 1980. Valle del Cauca (Santiago de Cali).

417. Lachesilla huitota Saenz, García Aldrete and González, 2015. Putumayo (Puerto Asís).

418. Lachesilla ilama García Aldrete, González and Saldaña, 2014. Valle del Cauca (Santiago de Cali).
419. L. ca. iguazuensis García Aldrete, 2004. Valle del Cauca (El Cerrito, Cerrito Adentro).

420. Lachesilla putumayensis Saenz, García Aldrete and González, 2015. Putumayo (Puerto Asís).

421. Lachesilla ca. rena Sommerman, 1946. Valle del Cauca (El Cerrito).

422. Lachesilla ca. teresiana García Aldrete and Mockford, 2009. Valle del Cauca (Santiago de Cali).

423. Lachesilla ca. veneper García Aldrete, 2010. Valle del Cauca (Santiago de Cali).

424. Lachesilla sp. 4. Valle del Cauca (Santiago de Cali).

425. Lachesilla sp. 5. Valle del Cauca (Santiago de Cali).

426. Lachesilla sp. 6. Valle del Cauca (Dagua).

427. Lachesilla sp. 7. Valle del Cauca (Dagua).

428. Lachesilla sp. 8. Valle del Cauca (Santiago de Cali).

429. Lachesilla sp. 9. Valle del Cauca (Santiago de Cali).

430. Lachesilla sp. 10. Valle del Cauca (Santiago de Cali).

431. Lachesilla sp. 11. Valle del Cauca (Dagua).

432. Lachesilla sp. 12. Valle del Cauca (Dagua).

433. Lachesilla sp. 13. Valle del Cauca (Yumbo).

434. Lachesilla sp. 14. Valle del Cauca (Tuluá).

435. Lachesilla sp. 15. Valle del Cauca (La Cumbre).

436. Lachesilla sp. 16. Valle del Cauca (Tuluá).

437. Lachesilla sp. 17. Valle del Cauca (Santiago de Cali).

438. Lachesilla sp. 18. Huila (NNP Cueva de Los Guácharos).

439. Lachesilla sp. 19. Huila (Palestina, La Mensura).

\section{Forcepeta species group}

440. Lachesilla ariasi García Aldrete, 2004. Putumayo (Puerto Asís).

441. Lachesilla asperiforceps García Aldrete, 2001. Valle del Cauca (Santiago de Cali).

442. Lachesilla bicornata New and Thornton, 1975. Valle del Cauca (Santiago de Cali).

443. Lachesilla ca. bicornata New and Thornton, 1975. Putumayo (Puerto Asís).

444. Lachesilla ecuatoriana García Aldrete, 2000. Valle del Cauca (El Cerrito).

445. Lachesilla ca. ecuatoriana García Aldrete. Valle del Cauca (Santiago de Cali).

446. Lachesilla falcata García Aldrete, 2000. Putumayo Puerto Asis). Amazonas (NNP Amacayacu).

447. Lachesilla pigmentithorax García Aldrete, 1996. Cauca (NNP Gorgona Island). Valle del Cauca (Santiago de Cali, Tuluá).

448. Lachesilla rugosa García Aldrete, 1996. Valle del Cauca (Darién, Santiago de Cali, La Cumbre, El Cerrito, Roldanillo).

449. Lachesilla valvula New and Thornton, 1975. Valle del Cauca (Roldanillo, Tuluá).

450. Lachesilla ca. valvula New and Thornton, 1975. Valle del Cauca (Roldanillo).

451. Lachesilla latiforceps García Aldrete and Mockford, 
2011. Valle del Cauca (Santiago de Cali).

452. Lachesilla ca. cuala García Aldrete, 1988. Valle del Cauca (Santiago de Cali).

453. Lachesilla ca. penta Sommerman, 1946. Valle del Cauca (Santiago de Cali).

454. Lachesilla ca. laciniosiforceps García Aldrete, 1999. Valle del Cauca (Darién).

455. Lachesilla ca. pigmentithorax García Aldrete, 1996. Valle del Cauca (El Cerrito).

456. Lachesilla sp. 6. Valle del Cauca (Santiago de Cali, El Cerrito).

457. Lachesilla sp. 7. Valle del Cauca (Santiago de Cali),

458. Lachesilla sp. 8. Valle del Cauca (Santiago de Cali).

459. Lachesilla sp. 9. Valle del Cauca (Buenaventura).

460. Lachesilla sp. 10. Putumayo (Puerto Asís).

461. Lachesilla sp. 11. Caquetá (San Vicente del Caguán).

\section{Riegeli species group}

462. Lachesilla riegeli Sommerman, 1946. Valle del Cauca (Dagua, Darién, Roldanillo).

463. Lachesilla ca. riegeli Sommerman, 1946. Valle del Cauca (Dagua).

\section{Sclera species group}

464. Lachesilla graminicola Badonnel, 1986. Valle del Cauca (Tenerife).

465. Lachesilla leonilae García Aldrete, 1986. Valle del Cauca (Darién, Santiago de Cali).

\section{Corona species group}

466. Lachesilla ca. picticeps Mockford, 1986. Valle del Cauca (La Cumbre, El Cerrito).

467. Lachesilla sp. 2. Valle del Cauca (Darién).

468. Lachesilla sp. 3. Valle del Cauca (Santiago de Cali).

469. Lachesilla sp. 4. Valle del Cauca (Santiago de Cali).

\section{Centralis species group}

470. Lachesilla ca. cintalapa García Aldrete, 1983. Valle del Cauca (El Cerrito).

471. Lachesilla ca. perezi García Aldrete, 1983. Valle del Cauca (Darién, Santiago de Cali).

\section{Genus Hemicaecilius Enderlein, 1903}

472. Hemicaecilius bogotanus Enderlein, 1903*. Cundinamarca (Bogotá).

473. Hemicaecilius mockfordi García Aldrete, González and Carrejo, 2012. Valle del Cauca (Santiago de Cali, El Cerrito, Sevilla).

474. Hemicaecilius smithersi García Aldrete, González and Carrejo, 2012. Valle del Cauca (Santiago de Cali).

475. Hemicaecilius nicolasi García Aldrete, González and Carrejo, 2012. Valle del Cauca (Santiago de Cali).

476. Hemicaecilius rauli García Aldrete, González and Carrejo, 2012. Valle del Cauca (Santiago de Cali).

477. Hemicaecilius sp. 5. Valle del Cauca (Dagua).
478. Hemicaecilius sp. 6. Norte de Santander (NNP Tamá).

Genus Nadleria Badonnel and García Aldrete, 1979

479. Nadleria sp. 1. Valle del Cauca (Dagua).

480. Nadleria sp. 2. Valle del Cauca (Dagua).

481. Nadleria sp. 3. Putumayo (Puerto Asís).

482. Nadleria sp. 4. Caquetá (San Vicente del Caguán).

\section{Genus Waoraniella García Aldrete, 2006}

483. Waoraniella andina Saenz, González and García Aldrete, 2018. Valle del Cauca (El Cerrito).

484. Waoraniella jarlinsoni Saenz, González and García Aldrete, 2018. Valle del Cauca (Santiago de Cali).

485. Waoraniella sp. 3. Huila (NNP Cueva de Los Guácharos).

\section{Subfamily Eolachesillinae \\ Tribe Graphocaeciliini}

Genus Acantholachesilla García Aldrete, Saenz and González, 2014

486. Acantholachesilla saltoensis García Aldrete, Saenz and González, 2014. Valle del Cauca (Buenaventura).

487. Acantholachesilla sp. Cauca (NNP Munchique).

\section{Genus Amazolachesilla García Aldrete and Mockford,} 2011

488. Amazolachesilla ca. ariasi García Aldrete and Mockford, 2011. Putumayo (Puerto Asís).

Genus Anomopsocus Roesler, 1940

489. Anomopsocus sp. A (see Mockford and Sullivan, 1986). Valle del Cauca (Santiago de Cali, Darién, Roldanillo).

\section{Genus Anomolachesilla García Aldrete, González and} Carrejo, 2012

490. Anomolachesilla palaciosi García Aldrete, González and Carrejo, 2012. Valle del Cauca (Santiago de Cali).

491. Anomolachesilla caldasiana García Aldrete, González and Carrejo, 2012. Caldas (Manizales).

\section{Genus Dagualachesilla García Aldrete, González and}

\section{Carrejo, 2013}

492. Dagualachesilla anchicayaensis García Aldrete, González and Carrejo, 2013. Valle del Cauca (Dagua, Buenaventura).

493. Dagualachesilla queremalensis García Aldrete, González and Carrejo, 2013. Valle del Cauca (Dagua, Buenaventura).

\section{Genus Dagualachesilloides García Aldrete, González and Carrejo, 2013}

494. Dagualachesilloides caliensis García Aldrete, González and Carrejo, 2013. Valle del Cauca (Santiago de Cali). 


\section{Genus Graphocaecilius Enderlein, 1900}

495. Graphocaecilius interpretatus Roesler, 1940. Valle del Cauca (Santiago de Cali)

496. Graphocaecilius mockfordi Sandoval, García Aldrete and González, 2016. Valle del Cauca (Santiago de Cali, La Cumbre). Risaralda (Planes de San Rafael Sanctuary).

497. Graphocaecilius peruvianus Mockford and Sullivan, 1986. Valle del Cauca (La Cumbre).

498. Graphocaecilius pictus Mockford and Sullivan, 1986. Boyacá (Moniquira).

499. Graphocaecilius sullivani Sandoval, García Aldrete and González, 2016. Valle del Cauca (Santiago de Cali).

500. Graphocaecilius turneri Sandoval, García Aldrete and González, 2016. Valle del Cauca (Santiago de Cali).

501. Graphocaecilius vartyi Turner, 1984. Valle del Cauca (Santiago de Cali, El Cerrito, Tenerife).

502. Graphocaecilius sp. 1. Huila (NR Meremberg).

503. Graphocaecilius sp. 2. Norte de Santander (NNP Tamá)

Genus Mesolachesilla Mockford and Sullivan, 1986

504. Mesolachesilla sp. 1. Valle del Cauca (Darién).

Genus Notolachesilla Mockford and Sullivan, 1986

505. Notolachesilla sp. 1. Valle del Cauca (El Cerrito).

Genus Prolachesilla Mockford and Sullivan, 1986

506. Prolachesilla sp. 1. Valle del Cauca (La Cumbre).

507. Prolachesilla sp. 2. Valle del Cauca (Santiago de Cali, La Cumbre, Darién).

508. Prolachesilla sp. 3. Valle del Cauca (Tenerife, El Cerrito).

509. Prolachesilla sp. 4. Caquetá (San Vicente del Caguán).

Genus Tricholachesilla Mockford and Sullivan, 1986

510. Tricholachesilla alata Mockford and Sullivan, 1986. Valle del Cauca (Buenaventura).

511. Tricholachesilla vampira Mockford and Sullivan, 1986. Chocó (NNP Los Katios).

512. Tricholachesilla sp. 1. Valle del Cauca (Buenaventura).

Genus Nanolachesilla Mockford and Sullivan, 1986

513. Nanolachesilla sp. Nariño (Santa Bárbara de Iscuandé).

Family Peripsocidae

Genus Kaestneriella Roesler, 1943

514. Kaestneriella ecuatoriana García Aldrete, 1989. Cauca (NNP Gorgona Island).

\section{Genus Peripsocus Hagen, 1866}

515. Peripsocus mutilatus Badonnel, 1986.* Cundinamarca (Páramo de Montserrate).
516. Peripsocus potosi Mockford, 1971*. Colombia. No locality given.

517. Peripsocus subfasciatus (Rambur, 1842)*.

Cundinamarca (ca. Bogotá, Bosque de Chicó).

518. Peripsocus terricolis Badonnel, 1986*.

Cundinamarca (Páramo de Montserrate).

519. Peripsocus stagnivagus Chapman, 1930. Cauca

(NN Gorgona Island). Valle del Cauca (Santiago de Cali).

520. Peripsocus sp. 1. Cauca (NNP Gorgona Island).

521. Peripsocus sp. 2. Valle del Cauca (El Cerrito, La Cumbre, Roldanillo, Santiago de Cali, Sevilla, Yumbo).

522. Peripsocus sp. 3. Valle del Cauca (Dagua, El Cerrito,

El Dovio, La Cumbre, Roldanillo, Santiago de Cali).

523. Peripsocus sp. 4. Valle del Cauca (Santiago de Cali).

524. Peripsocus sp. 5. Valle del Cauca (Dagua, Roldanillo).

525. Peripsocus sp. 6. Valle del Cauca (Yumbo).

526. Peripsocus sp. 7. Putumayo (Mocoa).

\section{Family Philotarsidae}

Genus Aaroniella Mockford, 1951

527. Aaroniella crista New and Thornton, 1975*. Cundinamarca (Bogotá).

528. Aaroniella sp. Cauca (NNP Gorgona Island).Valle del Cauca (Sevilla, Santiago de Cali).

\section{Genus Haplophallus Thornton, 1959}

529. Haplophallus leopardina (Williner, 1943)*. Cundinamarca (Páramo de Chisacá).

Genus Philotarsus Kolbe, 1880

530. Philotarsus thorntoni Turner, 1984*. Boyacá (Pozo Azul, Lago de Tota).

531. Philotarsus sp. 1. Norte de Santander (NNP Tamá).

Family Pseudocaeciliidae

Genus Allocaecilius Lee and Thornton, 1967

532. Allocaecilius sp. Valle del Cauca (Santiago de Cali).

Genus Scytopsocus Roesler, 1940

533. Scytopsocus sp. 1. Cauca (NNP Munchique). Huila (NR Meremberg). Norte de Santander (NNP Tamá).

534. Scytopsocus sp. 2. Huila (NR Meremberg).

Genus Heterocaecilius Lee and Thornton, 1967

535. Heterocaecilius sp. Cauca (NNP Gorgona Island).

Genus Pseudocaecilius Enderlein, 1903

536. Pseudocaecilius citricola (Ashmead, 1879). Cauca (NNP Gorgona Island).

\section{Infraorder Psocetae}

Family Hemipsocidae

Genus Hemipsocus Selys-Longchamps, 1872

537. Hemipsocus africanus Enderlein, 1907. Cauca (NNP 
Gorgona Island). Valle del Cauca (Santiago de Cali).

538. Hemipsocus chloroticus (Hagen, 1858). Valle del Cauca (La Cumbre).

\section{Family Psocidae}

Subfamily Amphigerontiinae

Tribe Amphigerontini

\section{Genus Amphigerontia Kolbe, 1880}

539. Amphigerontia alticola New and Thornton, 1975*. Cundinamarca (Bogotá).

540. Amphigerontia umbrata Navás, 1927*. "Columbia"

541. Amphigerontia sp. 1. Meta (Serranía de La Macarena, Resguardo Indígena Ukuae). Putumayo (Mocoa). Risaralda (Pueblo Rico, NR Río Negro). Valle del Cauca (Dagua, La Elsa).

542. Amphigerontia sp. 2. Boyacá. (FFS Iguaqué). Huila (NR Meremberg). Magdalena (NNP Sierra Nevada de Santa Marta). Norte de Santander (NNP Tamá, Herrán). Risaralda (Pueblo Rico, NR Río Negro).

543. Amphigerontia sp. 3. Cundinamarca (NNP Sumapaz).

544. Amphigerontia sp. 4. Cauca (Vereda Tijeras). Magdalena (San Javier).

545. Amphigerontia sp. 5. Cundinamarca (NR Chicaque, Tirolesa). Huila (NR Meremberg).

546. Amphigerontia sp. 6. Norte de Santander (NNP Tamá, Rafael Urdaneta).

547. Amphigerontia sp. 7. Risaralda (FFS OtúnQuimbaya).

548. Amphigerontia sp. 8. Norte de Santander (NNP Tamá, Rafael Urdaneta)

549. Amphigerontia sp. 9. Risaralda (FFS OtúnQuimbaya).

550. Amphigerontia sp. 10. Antioquia (San Roque).

551. Amphigerontia sp. 11. Chocó (NNP Los Katios).

\section{Genus Elaphopsocus Roesler, 1940}

552. Elaphopsocus gorrones Román-P., González and García Aldrete, 2015. Valle del Cauca (Dagua, Roldanillo, Santiago de Cali).

553. Elaphopsocus nasa Román-P., González and García Aldrete, 2015. Cauca (NNP Puracé).

554. Elaphopsocus rayoi Román-P., González and García Aldrete, 2015. Valle del Cauca (Roldanillo).

555. Elaphopsocus roesleri Román-P., González and García Aldrete, 2015. Huila (NR Meremberg).

556. Elaphopsocus roeslerioides Román-P., González and García Aldrete, 2015.Huila (RN Meremberg).

557. Elaphopsocus boyacaensis Román-P., González and García Aldrete, 2015. Boyacá (FFS Iguaqué).

558. Elaphopsocus cundinamarcaensis Román-P., González and García Aldrete, 2015.

Cundinamarca (NNP Chingaza).

Genus Elaphopsocoides Román-P., García Aldrete and González, 2014
559. Elaphopsocoides colombiensis Román-P., García Aldrete and González, 2014. Valle del Cauca (Santiago de Cali).

560. Elaphopsocoides lahonduraensis Román-P., García Aldrete and González, 2014. Valle del Cauca (El Dovio).

\section{Tribe Blastini}

\section{Genus Blaste Kolbe, 1883}

561. Blaste memorialis (Banks, 1920)*. Caldas.

562. Blaste sp. 1. Quindío (Filandia, Cañón del Río Barbas; Finca Agroforestal Bengala). Risaralda (FFS Otún-Quimbaya, El Molinillo). Valle del Cauca (Cerrito Adentro).

563. Blaste sp. 2. Meta (NNP Serranía de la Macarena, Caño Curía). Risaralda (FFS Otún-Quimbaya, Planes de San Rafael Sanctuary).

564. Blaste sp. 3. Amazonas (NNP Amacayacu). Caquetá (Solano, Resguardo Indígena Jericó-Consaya). Putumayo (Puerto Leguizamo, Resguardo Tukunare).

565. Blaste sp. 4. Amazonas (NNP Amacayacu). Caquetá (Solano, Resguardo Indígena Jericó-Consaya). Putumayo (Puerto Asís).

566. Blaste sp. 5. Putumayo (Puerto Asís).

567. Blaste sp. 6. Cauca (NNP Munchique, NNP Gorgona Island). Huila (NR Meremberg). Magdalena (San Javier). Risaralda (FFS Otún-Quimbaya). Valle del Cauca (NNP Farallones de Cali).

568. Blaste sp. 7. Risaralda (FFS Otún-Quimbaya). Putumayo (Puerto Asís).

569. Blaste sp. 8. Cauca (NNP Gorgona Island).

570. Blaste sp. 9. Norte de Santander (NNP Tamá, Herrán).

571. Blaste sp. 10. Huila (NNP Cueva de los Guácharos, NR Meremberg). Norte de Santander (NNP Tamá).

\section{Genus Blastopsocus Roesler, 1943}

572. Blastopsocus mockfordi Badonnel, 1986*. Cundinamarca (Ca. Bogotá, between Subachoque and Páramo El Tablazo). Boyacá (FFS Iguaqué).

573. Blastopsocus sp. 1. Risaralda (FFS Otún-Quimbaya, Pueblo Rico).

574. Blastopsocus sp. 2. Magdalena (NNP Sierra Nevada de Santa Marta). Valle del Cauca (NNP Farallones de Cali, La Meseta, Roldanillo, Montañuela).

575. Blastopsocus sp. 3. Magdalena (NNP Sierra Nevada de Santa Marta). Norte de Santander (NNP Tamá, Herrán).

576. Blastopsocus sp. 4. Putumayo (Puerto Leguizamo, Resguardo Tukunare).

577. Blastopsocus sp. 5. Magdalena (San Javier).

578. Blastopsocus sp. 6. Boyacá (FFS Iguaqué). Cundinamarca. Valle del Cauca (Santiago de Cali).

579. Blastopsocus sp. 7. Chocó (NNP Los Katios).

580. Blastopsocus sp. 8. Caquetá (Belén de los Andaquíes). 


\section{Genus Chaetoblaste García Aldrete and Román-P.,} 2015

581. Chaetoblaste gonzalezi García Aldrete and Román-P., 2015. Valle del Cauca (Santiago de Cali).

582. Chaetoblaste sp. 1. Amazonas (NNP Amacayacu).

583. Chaetoblaste sp. 2. Putumayo (Puerto Asís, Puerto Leguizamo).

584. Chaetoblaste sp. 3. Huila (NNP Cueva de los Guácharos, NR Meremberg).

585. Chaetoblaste sp. 4. Huila (NNP Cueva de los Guácharos).

586. Chaetoblaste sp. 5. Caquetá (Belén de los Andaquíes).

\section{Genus Chaetopsocidus Badonnel, 1986}

587. Chaetopsocidus sturmi Badonnel, 1986*. Cundinamarca. (Páramo de Montserrate).

\section{Subfamiy Psocinae Tribe Ptyctini}

Genus Indiopsocus Mockford, 1974

588. Indiopsocus abouchaari (Badonnel, 1986)*. Cundinamarca (Subpáramo de Montserrate).

589. Indiopsocus expansus (New and Thornton, 1975)*. Cundinamarca (Bogotá).

590. Indiopsocus fittkaui (Badonnel, 1986)*. Cundinamarca (Páramo de Montserrate).

591. Indiopsocus sp. 1. Cauca (NNP Gorgona Island).

592. Indiopsocus sp. 2. Cauca (NNP Gorgona Island).

\section{Genus Oreopsocus Roesler, 1939}

593. Oreopsocus sp. 1. Amazonas (NNP Amacayacu). Risaralda (FFS Otún- Quimbaya, Pueblo Rico).

594. Oreopsocus sp. 2. Magdalena (San Javier). Risaralda (FFS Otún-Quimbaya).

\section{Genus Psocomesites Roesler, 1943}

595. Psocomesites sturmi Badonnel, 1986*. Cundinamarca (Páramo de Montserrate).

596. Psocomesites sp. 1. Norte de Santander (NNP Tamá).

597. Psocomesites sp. 2. Cauca (Tijeras).

598. Psocomesites sp. 3. Huila (NR Meremberg).

\section{Genus Ptycta Enderlein, 1925}

599. Ptycta sp. 1. Risaralda (Pueblo Rico).

600. Ptycta sp. 2. Risaralda (Pueblo Rico). Valle del Cauca (Roldanillo).

601. Ptycta sp. 3. Risaralda (Planes de San Rafael Sanctuary).

602. Ptycta sp. 4. Huila (NR Meremberg). Quindío (Filandia).

603. Ptycta sp. 5. Magdalena (NNP Sierra Nevada de Santa Marta).

604. Ptycta sp. 6. Boyacá (FFS Iguaqué). Cauca (NNP Gorgona Island).

605. Ptycta sp. 7. Boyacá (FFS Iguaqué).
606. Ptycta sp. 8. Risaralda (FFS Otún-Quimbaya).

607. Ptycta sp. 9. Norte de Santander (NNP Tamá).

608. Ptycta sp. 10. Amazonas (NNP Amacayacu). Boyacá FFS Iguaqué).

609. Ptycta sp. 11. Putumayo (Puerto Asís).

610. Ptycta sp. 12. Huila (NR Meremberg).

611. Ptycta sp. 13. Norte de Santander (NNP Tamá).

612. Ptycta sp. 14. Caldas (NNP Nevado del Ruiz).

613. Ptycta sp. 15. Norte de Santander (NNP Tamá, Toledo).

614. Ptycta sp. 16. Caquetá (Belén de los Andaquíes).

\section{Genus Steleops Enderlein, 1910}

615. Steleops buitrerensis González, García Aldrete and Carrejo, 2011. Valle del Cauca (Dagua, Santiago de Cali).

616. Steleops manizalensis González, García Aldrete and Carrejo, 2011b. Caldas (Manizales).

617. Steleops pulcher New, 1972. Valle del Cauca (Tuluá).

618. Steleops rioblancoensis González, García Aldrete and Carrejo, 2011b. Caldas (Manizales).

619. Steleops mendivili González, García Aldrete and Carrejo, 2011b. Valle del Cauca (Dagua). Risaralda (Pueblo Rico, NR Río Negro).

620. Steleops plenitudensis González, García Aldrete and Carrejo, 2011b. Valle del Cauca (Dagua).

621. Steleops sp. 1. Valle del Cauca (La Cumbre).

622. Steleops sp. 2. Valle del Cauca (Cali, San Antonio).

623. Steleops sp. 3. Cauca (Coconuco).

624. Steleops sp. 4. Valle del Cauca (La Cumbre).

625. Steleops sp. 5. Valle del Cauca (Quebrada Honda).

626. Steleops sp. 6. Putumayo (Puerto Asís).

627. Steleops sp. 7. Putumayo (Puerto Asís).

628. Steleops sp. 8. Boyacá (FFS Iguaqué).

629. Steleops sp. 9. Huila (NR Meremberg).

630. Steleops sp. 10. Huila (NR Meremberg).

631. Steleops sp. 11. Valle del Cauca (Dagua, Km. 38).

632. Steleops sp. 12. Valle del Cauca (Obando).

633. Steleops sp. 13. Caquetá (San Vicente del Caguán).

\section{Genus Trichadenotecnum Enderlein, 1909}

634. Trichadenotecnum decui Badonnel, 1987. Magdalena (San Javier). Risaralda (Pueblo Rico, NR Río Negro).

635. Trichadenotecnum bos Yoshizawa, García Aldrete and Mockford, 2008. Putumayo (Puerto Asís).

636. Trichadenotecnum peruense Yoshizawa, García Aldrete and Mockford. 2008. Huila (NR Meremberg). Putumayo (Puerto Asís).

637. Trichadenotecnum slossonae (Banks, 1903). Putumayo (Mocoa).

638. Trichadenotecnum sp. 1. Huila (NR Meremberg). Risaralda (Pueblo Rico, NR Río Negro).

639. Trichadenotecnum sp. 2. Antioquia (San Roque). 


\section{Tribe Metylophorini}

Genus Brachinodiscus Enderlein, 1925

640. Brachinodiscus sp. 1. Risaralda (Planes de San Rafael Sanctuary). Huila (NR Meremberg).

641. Brachinodiscus sp. 2. Risaralda (Planes de San Rafael Sanctuary). Huila (NR Meremberg).

642. Brachinodiscus $\mathrm{sp}$. 3. Boyacá (FFS Iguaqué). Huila (NR Meremberg, NNP Cueva de los Guácharos).

\section{Genus Metylophorus Pearman, 1932}

643. Metylophorus sp. 1. Risaralda (Planes de San Rafael Sanctuary).

644. Metylophorus sp. 2. Boyacá (FFS Iguaqué). Huila (NNP Cueva de los Guácharos). Norte de Santander (NNP Tamá, Rafael Urdaneta).

645. Metylophorus sp. 3. Boyacá (FFS Iguaqué). Risaralda (FFS Otún-Quimbaya).

646. Metylophorus sp. 4. Boyacá (FFS Iguaqué). Huila (NR Meremberg). Valle del Cauca (Roldanillo).

647. Metylophorus sp. 5. Valle del Cauca (NNP Farallones, Jamundi, La Meseta).

648. Metylophorus sp. 6. Huila (NR Meremberg). Norte de Santander (NNP Tamá, Rafael Urdaneta).

649. Metylophorus sp. 7. Norte de Santander (NNP Tamá).

\section{Tribe Psocini}

\section{Genus Psocus Latreille, 1794}

650. Psocus sp. 1. Amazonas (NNP Amacayacu). Putumayo (Mocoa, Puerto Asís).

651. Psocus sp. 2. Amazonas (NNP Amacayacu).

652. Psocus sp. 3. Risaralda (FFS Otún-Quimbaya). Putumayo (Puerto Asís).

653. Psocus sp. 4. Amazonas (NNP Amacayacu).

654. Psocus sp. 5. Norte de Santander (NNP Tamá). Putumayo (Puerto Leguizamo).

655. Psocus sp. 6. Norte de Santander (NNP Tamá, Herrán).

656. Psocus sp. 7. Huila (NR Meremberg).

657. Psocus sp. 8. Caquetá (Belén de los Andaquíes).

\section{Tribe Thyrsophorini}

\section{Genus Cerastipsocus Kolbe, 1884}

658. Cerastipsocus bogotanus (Kolbe, 1883)*. Colombia.

659. Cerastipsocus coloratus (Kolbe, 1883)*. Colombia.

660. Cerastipsocus infectus (McLachlan, 1886)*. Colombia.

661. Cerastipsocus moestus (Kolbe, 1883)*. Colombia.

662. Cerastipsocus pallidinervis (Kolbe, 1883)*. Colombia.

663. Cerastipsocus vetustus (Kolbe, 1883)*. Colombia.

664. Cerastipsocus trifasciatus (Provancher, 1876). Valle del Cauca (Dagua, Santiago de Cali).

665. Cerastipsocus sp. 1. Amazonas (NNP Amacayacu). Caquetá (Solano)

Magdalena

(NNP

Tayrona).
666. Cerastipsocus sp. 2. Boyacá (FFS Iguaqué). Putumayo (Puerto Asís).

667. Cerastipsocus sp. 3. Cundinamarca (NNP Chingaza, Bogotá: Humedal La Conejera).

668. Cerastipsocus sp. 4. Valle del Cauca (NNP Farallones, Jamundí).

669. Cerastipsocus sp. 5. Norte de Santander (NNP Tamá, Herrán).

670. Cerastipsocus sp. 6. Putumayo (Puerto Leguizamo).

671. Cerastipsocus sp. 7. Putumayo (Puerto Leguizamo). Caquetá (Belén de Los Andaquíes).

672. Cerastipsocus sp. 8. Huila (NR Meremberg).

673. Cerastipsocus sp. 9. Valle del Cauca (Santiago de Cali).

\section{Genus Cervopsocus New, 1978}

674. Cervopsocus medialis New, 1978. Huila (Palestina, NR El Encanto).

\section{Genus Psococerastis Pearman, 1932}

675. Psococerastis opulenta (Navás, 1930). Chocó (Pangala).

676. Psococerastis pyralinella Mockford, 1981. Magdalena (Sierra de Perija, Socorpa Mission).

677. Psococerastis sp. 1. Amazonas (NNP Amacayacu).

678. Psococerastis sp. 2. Risaralda (FFS OtúnQuimbaya). Valle del Cauca (NNP Farallones, Jamundí).

679. Psococerastis sp. 3. Valle del Cauca (NNPFarallones, Jamundí). Vaupés (Mosiro-Itajura Biological Station, Caparú).

680. Psococerastis sp. 4. Boyacá (FFS Iguaqué). Cauca (NNP Gorgona Island). Risaralda (FFS OtúnQuimbaya).

681. Psococerastis sp. 5. Boyacá (FFS Iguaqué). Nariño (NR La Planada). Valle del Cauca (NNP Farallones, Jamundí, La Meseta).

682. Psococerastis sp. 6. Magdalena (NNP Tayrona).

683. Psococerastis sp. 7. Amazonas (Leticia, Forest Reserve Río Calderón). Caquetá ( $\quad \mathrm{N} \quad \mathrm{N} \quad \mathrm{P}$ Chibiriquete).

684. Psococerastis sp. 8. Boyacá (FFS Iguaqué). Huila (NNP Cueva de los Guácharos).

685. Psococerastis sp. 9. Risaralda (FFS OtúnQuimbaya).

686. Psococerastis sp. 10. Meta (NNP Serranía de la Macarena, Resguardo Ukuae).

687. Psococerastis sp. 11. Caquetá (Solano, JericóConsaya).

Genus Thyrsophorus Burmeister, 1839

688. Thyrsophorus sp. 1. Meta (NNP Serranía de la Macarena, Caño Curía).

\section{Genus Thyrsopsocopsis Mockford, 2004}

689. Thyrsopsocopsis sp. 1. Vaupés.

690. Thyrsopsocopsis sp. 2 Risaralda (FFS Otún- 
Quimbaya).

691. Thyrsopsocopsis sp. 3 Putumayo (NNP La Paya).

Family Myopsocidae

Genus Lichenomima Enderlein, 1910

692. Lichenomina onca Mockford, 1991. Valle del Cauca (Santiago de Cali).

693. Lichenomina sp. 1. Cauca (NNP Gorgona Island). Caquetá (Belén de los Andaquíes).

694. Lichenomina sp. 2. Cauca (NNP Gorgona Island).

695. Lichenomina sp. 3. Valle del Cauca (Roldanillo, Dagua, El Dovio.)

696. Lichenomina sp. 4. Valle del Cauca (Santiago de Cali, San Antonio).

697. Lichenomina sp. 5. Risaralda (Pueblo Rico, NR Río Negro).

698. Lichenomina sp. 6. Valle del Cauca (Buenaventura, Pericos).

699. Lichenomina sp. 7. Cauca (NNP Gorgona Island).

700. Lichenomina sp. 8. Nariño (Santa Barbara de Iscuandé).

701. Lichenomina sp. 9. Amazonas (NNP Amacayacu). Meta (Serranía de La Macarena, Resguardo Indígena Kiwe).

702. Lichenomina sp. 10. Amazonas (NNP Amacayacu).

703. Lichenomina sp. 11. Chocó (NNP Los Katios).

\section{Genus Myopsocus Hagen, 1866}

704. Myopsocus minor Hagen, 1866. Amazonas (NNP Amacayacu).

705. Myopsocus minutus (Mockford, 1974). Cauca (NNP Gorgona Island). Nariño (Santa Bárbara de Iscuandé).

706. Myopsocus sp. 1. Cauca (NNP Gorgona Island). Valle del Cauca (El Cerrito).

707. Myopsocus sp. 2. Amazonas (NNP Amacayacu).

708. Myopsocus sp. 3. Valle del Cauca (Santiago de Cali).

709. Myopsocus sp. 4. Valle del Cauca (Santiago de Cali).

710. Myopsocus sp. 5. Chocó (NNP Los Katios).

711. Myopsocus sp. 6. Putumayo (Puerto Leguizamo).

712. Myopsocus sp. 7. Chocó (NNP Los Katios). Nariño (Santa Bárbara de Iscuandé).

713. Myopsocus sp. 8. Amazonas (NNP Amacayacu). Meta (Sierra de La Macarena).

714. Myopsocus sp. 9. Amazonas (NNP Amacayacu).

715. Myopsocus sp. 10. Chocó (NNP Los Katios). Putumayo (Mocoa, Puerto Asís).

716. Myopsocus sp. 11. Caquetá (Belén de los Andaquíes).

717. Myopsocus sp. 12. Caquetá (Belén de los Andaquíes).

718. Myopsocus sp. 13. Chocó (NNP Los Katios).

719. Myopsocus sp. 14. Putumayo (Puerto Leguizamo).

720. Myopsocus sp. 15. Caquetá (Belén de los Andaquíes). Putumayo (Mocoa).

\section{KEY TO FAMILIES AND SUBFAMILIES OF PSOCODEA: 'PSOCOPTERA' FROM COLOMBIA}

Adapted from García Aldrete and Mockford (2012)

1. Adults with more than 18 flagellomeres; hypopharyngeal filaments separate their entire length, never fused on midline; labial palpus with minute basal segment and rounded distal segment Suborder TROGIOMORPHA .. 3

1'. Adults with fewer than 18 flagellomeres (usually 11-13); hypopharyngeal filaments fused along midline at least part of their length; labial palpus as above, or with no basal segment .................. 2

2(1'). Adults with 13 flagellomeres (rarely fewer); at least some flagellomeres annulated with cuticular sculpture. Labial palpus usually with a minute basal segment and a rounded distal segment. Tarsi usually three-segmented. Forewing, when present, lacking sclerotized pterostigma (Figs 3 A-G) ....... Suborder TROCTOMORPHA .... 6

2'. Adults with 11 flagellomeres (rarely fewer); no flagellomeres annulated with cuticular sculpture but sometimes with reticulate sculpture in cuticle. Labial palpus lacking basal segment, consisting of a single rounded or triangular segment. Tarsi two-or three-segmented. Forewings, when fully developed, with sclerotized pterostigma (Figs 3HL, 4A-L, 5A-L)

Forewings, when fully developed, with vein $\mathrm{Cu} 2(\mathrm{CuP})$ and $1 \mathrm{~A}$ ending separately on wing margin (Fig. 3B); spur sensillum always present on Mx2. Ovipositor valvulae: v3 elongate, partially joined together on midline by membrane; v2 small or absent; vl absent Infraorder Atropetae .... 4

3'. Forewings, when fully developed, with veins $\mathrm{Cu} 2$ $(\mathrm{CuP})$ and $1 \mathrm{~A}$ ending together on wing margin (point of junction called 'nodulus') (Fig. 3A); spur sensillum of $\mathrm{Mx} 2$ present or absent. Ovipositor valvulae: v3 never elongate, but sometimes very broad; v2 usually present, slender; v1 frequently present, slender. Infraorder

Psocatropetae Psyllipsocidae

4(3). Body and forewings covered with scales or dense setae. Wings often pointed apically, with visible veins (Fig. 3B). Mesocoxae of the two sides capable of interlocking by a 'button and cavity' mechanism ........................ Lepidopsocidae .... 5

4' Body and forewings never covered with scales or dense setae. Wings not pointed apically, with visible veins. Mesocoxae of the two sides not capable of interlocking .................... Psoquillidae

5. Forewings and body clothed with scales. Antenna with $<20$ flagellomeres, the basal ones beyond $\mathrm{fl}$ much more than 3 times as 
long as wide. Hindwings with a closed cell Perientominae

5'. Antennae with 30 or more flagellomeres, the basal segments beyond $\mathrm{fl}$ ea. 2 times as long as wide. Hindwings lacking a closed cell

Lepidopsocinae

6(2). Small forms, rarely over $2 \mathrm{~mm}$ in length. Males wingless, and many females wingless or brachypterous. Forewings of macropterous females with not more than two $\mathrm{M}$ branches and lacking vein IIA (Fig. 3E). Pearman's coxal organ absent or represented by a slight bulge in cuticle .. Infraorden Nanopsocetae .......7

6'. Body size variable but usually at least $3 \mathrm{~mm}$ in length. Wings present in adults of both sexes, forewings with $\mathrm{M}$ three-branched and vein IIA usually present (Fig. 3C). Pearman's coxal organ represented by a mirror and usually a rasp Infraorden Amphientometae ........9

7(6). Body flattened; coxae of opposite sides widely separated by broad sternal plates. Forewings, when present (some females) with two parallel longitudinal veins occupying main body of wing......

Liposcelididae .....8

7'. Body not flattened; coxae of opposite sides only narrowly separated. Forewings, when present (some females), with several branching veins occupying main body of wing (Fig. 3E) Pachytroctidae

8(7) Hind femur with a lateral protuberance. Females apterous, with a T-shaped sclerite on the subgenital plate........................... Liposcelidinae

8' Hind femur lacking a lateral protuberance. Females apterous or macropterous, rarely brachypterous, lacking a T-shaped sclerite on the subgenital plate. Embidopsocinae

$9\left(6^{\prime}\right)$ Body and forewings densely covered with scales. In forewing veins IA and IIA separate their entire length (Fig. 3C) . Amphientomidae

9' Body and forewings lacking scales. In forewing veinsIAandIIAjoined togetherbeforereachingwing margin (Figs 3D, F, G) . Electrentomoidea .... 10

10(9') Pterostigma open basally (Fig. 3F, 3G). Front tibia with a distal brush of stout setae on inner surface 11

10' Pterostigma closed basally (Fig. 3D). Front tibia lackingadistalbrushofstoutsetaeoninnersurface.12

11(10) Forewing with a long Rs-M crossvein (Fig. 3G); forewings unpigmented. Outer cusp of lacinial tip with only two denticles. Musapsocidae

11' $\mathrm{Rs}$ and $\mathrm{M}$ in forewing joined at a point (Fig. 3F); forewings with cloudy pigmentation throughout or with distinct spotting and banding pattern. Outer cusp of lacinial tip with three denticles .. Troctopsocidae

12(10') Pretarsal claw with a single preapical denticle. V3, where known, only shallowly, or not at all bilobed .. Electrentomidae

12' Pretarsal claw with two preapical denticles. V3 deeply bilobed .. Compsocidae

13(2') Meso-precoxal bridges narrow and corresponding mesotrochantins broad basally. Labrum curved on sides, well molded to contours of mandibles. .. Infraorder Psocetae . 16

13' Meso-precoxal bridges and mesotrochantins not as above (i.e. the former wide and the latter narrow basally), or, if otherwise, labrum broad and flat, bearing a longitudinal pair of sclerites. ........ 14

14(13') Pretarsal claw lacking preapical denticle .................... Infraorder Caeciliusetae .......... 19

14 Pretarsal claw usually with a preapical denticle; if denticle absent (Family Archipsocidae) forewings and body are densely setose. 15

15. V3 largely fused to v2. Labrum with a pair of longitudinal sutures running through it (Fig. 1). Labrum usually flat, not conforming closely to contours of mandibles; mandibles elongate Infraorder Epipsocetae ..... 24

15 V3 largely free from v2 and from body wall. Labrum curved on sides, well molded to contours of mandibles, lacking a pair of longitudinal sutures running through it .InfraorderHomilopsocidea.....27

16(13) Vein Minforewing two-branched, the Mstemjoined to vein $\mathrm{CuA} 1$ by a long crossvein (Fig. $3 \mathrm{H})$; hind tibia lacking longitudinal row of ctenidiobothria

Hemipsocidae

16' Vein $M$ in forewing three-branched, the $M$ stem usually fused for a short distance to vein $\mathrm{CuA} 1$; rarely the two joined by a short crossvein (Figs 3I-L). Hind tibia with a longitudinal row of ctenidiobothria ............... 17

17(16') Tarsi three-segmented; forewings heavily blotched with brown, the margins with altemating brown and colorless banding (Fig. 3I) ............ Myopsocidae

17'. Tarsi two-segmented; forewing markings variable (Figs 3J-L), but margins not with altemating brown and colorless banding ....... Psocidae ...... 18

$18\left(17^{\prime}\right)$. Males with parameres distally separated. Hypandrium resulting from the fusion of sternites VIII and IX. Paraprocts with rounded processes. Females with v2 bearing a thin and conical process apically ................................ Amphigerontiinae

18' Males with phallosome closed. Hypandrium confined to IX sternum, and a pair of median sclerites or a thin band in VIII sternite ...... Psocinae

19(14) Ventral abdominal vesicles absent. Mesepistemal sulcus absent. Mandibles short to moderate in length. Forewing with vein $M$ twobranched (Fig. 5I) .................. Asiopsocidae

19' With one to three ventral abdominal vesicles. Mesepisternal sulcus present. Mandibles elongate. Forewing with vein $\mathrm{M}$ two or three-branched (Figs 
4G-K) ............. Superfamily Caeciliusoidea ... 20

20(19') Forewings with a crossvein from the pterostigma to Rs stem and another from M stem to areola postica (Fig. 4J) Stenopsocidae

20' Forewing lacking the crossveins indicated above (Figs 4G, H, K) .. 21

21(20') Ciliation of hindwing margin restricted to cell $\mathrm{r} 3$ or none. $\mathrm{Mx} 4$ longer than $\mathrm{Mx} 2$. Spine of free margin of paraproct large.....

Dasydemellidae

21' Ciliation of hindwing margin complete except for basal two-thirds of front margin (Figs 4G, H). Mx4 shorter than or equal to Mx2. Spine of free margin of paraproct small or absent. ........ 22

22(21') Setae of veins in distal half of forewing on both dorsal and ventral surfaces, vein M 2 or 3-branched (Fig. 4H). Mx4=Mx2 in length .... Amphipsocidae

22. Setae of veins in distal half of forewing only on dorsal surface, vein M 3-branched (Fig. 4G). Mx4 shorterthan Mx2 ................. Caeciliusidae......23

23(22') With three ventral abdominal vesicles and seven distal inner labral sensilla ............ Paracaeciliinae

23' With two (rarely one) ventral abdominal vesicles and five distal inner labral sensilla

Caeciliusinae

24(15) Forewings with one anal vein, pterostigma with or without crossveins (Figs. 4A-D); some species with brachypterousomicropterous forms... Epipsocidae

24'. Forewings with two anal veins (Figs. 4I, L), pterostigma without crossveins; some families with brachypterous or apterous forms ................. 25

25(24) Lacinial tip with outer cusp slender, bearing not more than three denticles. Pretarsal claw with pulvillus bent near base, expanded at tip. Forewing as in figures 4I, L) ................... Dolabellopsocidae

25. Lacinial tip with outer cusp broader, bearing more than three denticles. Pretarsal claw with pulvillus straight and pointed distally. Forewing as in figures 4E-F,5A-D). . .26

26(25') Pretarsal claw with a basal spine in addition to the more distal pulvillus. Forewings with or without spur veins (Figs 4E, 4F), M with three branchs. The two labral sutures reaching proximal margin of labrum and curving around to lateral margin (Fig 1A)

Cladiopsocidae

26' Pretarsal claw with only the spine-like pulvillus. Forewing veins lacking spur veins, $\mathrm{M}$ with threenine branches (Figs 5A-D). The two labral sutures never reaching proximal margin of labrum (Fig. 1B), except in Willreevesia García Aldrete .....

Ptiloneuridae

$27\left(15^{\prime}\right)$. In forewings, setae of wing margin in m cells in two series forming crossing pairs (Figs 5E-G). Brachypterous and micropterous individuals with numerous long, backward-directed setae on vertex and thoracic dorsum .......................................... 28

27 Forewing margin lacking series of crossing hairs
(Figs 5H-L). Brachypterous and micropterous individuals with at most only moderate body ciliation ......................................................... 32

28(27) Venation in forewing vague; Rs in forewing often unbranched (Fig. 5E). Wings often greatly reduced; in both macropterous and short-winged forms numerous long, backward directed setae on vertex and thoracic dorsum. Usually colonial forms living under dense webbing

Archipsocidae .... 29

28 Venation in forewing distinct; Rs in forewing with two branches. Wings usually not reduced. Vertex and thoracic dorsum lacking numerous long, backward-directed setae. Generally solitary, or a few individuals living together, either in open or under light webbing ....................... 30

29(28) Species with frequent neoteny: males always micropterous or apterous, females often brachypterous or micropterous. Hindwing with a closed cell Archipsocinae

29' Species lacking neoteny, both sexes macropterous. Hindwing usually lacking a closed cell .Parachipsocinae

30(28') Adults with tarsi two-segmented. External parameres generally much longer than aedeagal arch. Forewings with or without setae on the membrane (5F). Leaf-inhabiting forms

30, ...................................Pseudocaeciliidae Adults usually with three tarsal segments. External parameres only slightly longer or shorter than aedeagal arch. Forewing as in figure 3G. Barkinhabiting forms

. Philotarsidae..... 31

31(30') Apex of terminal antennomere not produced, not bearing a single apical seta. Vein $\mathrm{Cul}(\mathrm{CuA})$ in hindwing setose

Philotarsinae

31' Apex of terminal antennomere produced, bearing a single apical seta. Vein $\mathrm{Cul}$ in hindwing bare ...

Aaroniellinae

32(27') Macropterous forms ....................................... 33

32' Micropterous or apterous forms ………............ 37

33(32) Forewing with vein CuA1 present and usually free from M (Figs 5J-K). Wings either unciliated or lightly ciliated. Ovipositor reduced to one (v3), rarely two valvulae on each side Lachesillidae ..... 34

33 Forewing lacking vein $\mathrm{CuA1}$ (Figs 5H, L). Wings lightly to moderately ciliated. Ovipositor usually with three valvulae, rarely reduced to one on each

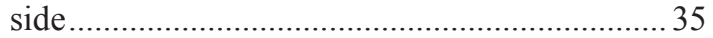

34(33) Tarsi two- or three segmented. Forewings sparsely ciliated on veins (Fig. 5J) and margins, membrane of pterostigma ciliated or not. Phallosome a closed frame, endophallic sclerites variable in size, some chelate. V3 elongate, tapering distally; setae primarily along outer margin. Female paraprocts with field of short, 
stout setae along median margin in ventral half Eolachesillinae

34' Tarsi two segmented. Forewings with ciliation on veins, margins, and sparsely on membrane or only on veins and margins, sometimes very sparse and short (Fig. 5K). Phallosome open distally, variable. Endophallus apparently absent. V3 variable in shape; setae, when present, distributed over its entire surface. Female paraprocts without field of short, stout setae along median margin in ventral half ................................................ Lachesillinae

35(33') Vein R1 in pterostigma parallel to wing margin most of its length (Fig. 5H) Ectopsocidae

35 $\quad$ Vein R1 in pterostigma curved (Fig. 5L) . 36.

36(35') Both sexes macropterous (Fig. 5L). Phallosome elongate, with heavily sclerotized endophallic structuredistallyinformofathree-prongedfork.

Peripsocidae

36 Either males or females macropterous, not both. Endophallusnotasabove.

Elipsocidae

37(32) Epistomal suture present dorsally, separating frons from postc 1ypeus. Subgenital plate usually with two distalprocesses.

Ectopsocidae

37 Epistomal suture absent dorsally, postclypeus and frons continuous. Subgenital plate with a single, relatively broad median distal process

Peripsocidae

\section{DISCUSSION}

Up to 2010, psocodeans were among the least studied of Colombian insects. The results presented here, derived mostly from studies carried out from 2010 to date, raise the number of psocids recorded in Colombia from 79 (Lienhard and Smithers 2002), to 720 species. The number of psocid species recorded in Colombia is therefore increased by $811.4 \%$. Below, we present a brief analysis of these results, with special focus on the studies conducted by our research group.

Recent estimates of the species diversity of 'Psocoptera' indicate that 5,732 species have been described so far (Zhang, 2013). However, these numbers could easily raise to more than 10,000 species if the neotropical diversity is included. Lienhard and Smithers (2002) recorded for South America about 762 species, of which 425 species were Brazilian (García Aldrete \& Mockford, 2009). The same authors also suggested that the number of species recorded in Brazil, and in general for South America is highly underestimated. The Brazilian psocid fauna actually consists of 618 species [see Silva Neto et al. (2014); Silva Neto \& García Aldrete (2016)].This trend is thought to be directly related to (i) the small amount of taxonomic work conducted in the area, (ii) the reduced number of specialists, and (iii) the non-extensive collections conducted in the area. Colombia is considered to be a megadiverse country, the few records published until 2002 indicate that the psocid diversity was underestimated (i.e., 79 species; see Lienhard and Smithers, 2002; Lienhard, 2004). Of the 720 psocid especies listed here, 426 remain to be described, and about 200 are still undefined. The latter category means that we are not actually confident on whether these species have been described or not, but they are probably new to science.

The diversity found in Colombia is comparable in number of species to that of Mexico. In the latter country, over 50 years of field work and numerous studies indicate that the psocid fauna is represented by about 766 species (García Aldrete, 2014). Thus, based on the estimates presented in this study, Colombia is the most species rich country in South, Central and North America, with about $12.5 \%$ of the psocid world fauna, with a high level of endemism. The results presented here demonstrate that 'Psocoptera' had been almost ignored from the scientific research of the country.

We acknowledge that sampling representativity in this study is not enough to conclude on the total number of species occurring in Colombia. Given that the sampled localities correspond to a small part of the 59 natural areas belonging to the System of Colombian National Natural Parks, psocid richness might be much higher than the one listed here. In addition, collection bias might have also contribuited to the underestimation of the psocopteran richness in the country (i.e., only Malaise traps were used to collect samples deposited in the Alexander von Humboldt Institute).

Six of the 28 families recorded in Colombia represent $83.8 \%$ of the species found (i.e., Cladiopsocidae, Epipsocidae, Ptiloneuridae, Lachesillidae, Psocidae and Myopsocidae). At least 426 new species were identified in the six families mentioned above: $92.6 \%$ of new species for Cladiopsocidae, $89.4 \%$ for Epipsocidae, $66 \%$ for Lachesillidae, and $89.2 \%$ for Ptiloneuridae. Interestingly, the latter family was only known from a single Colombian species, but it now includes 141 species. This richness is unevenly distributed in the genera Triplocania (52 species), Loneura (38 species), Euplocania (36 species), and Loneuroides (7 species). Also, species known in Peru and Brazil were found in Colombia, such as Perucania longiareola, Euplocania badonneli, Ptiloneura bidorsalis, Triplocania erwini and Triplocania lamasi.

While a single Cladiopsocus species was known in Colombia by 2004, we raised this number to 27 species. Calderón et al. (2014) recorded six species, but additional collecting, mostly in the Amazonian piedmont, allowed the discovery of 21 species, eight of which have been recently described (González et al. 2016), and 13 species are in process of publication. This raises the genus richness to 41 species, 39 of which are exclusively neotropical, with Colombia being the most species rich country for this genus.

148 species of Epipsocidae, in 11 genera, were found in this work. Epipsocus Hagen, Goja Navás and Gojaoides García Aldrete group $70.5 \%$ of the species found. About 
$90 \%$ of the Colombian species are undescribed, and two new genera have been erected (García Aldrete et al. 2015).

Psocidae is the largest family of 'Psocoptera', including 65 genera, 20 of which were found in Colombia. For some genera, a significant increase in the number of species was found, for instance, the genus Elaphopsocus Roesler, described 77 years ago, was known from a single Brazilian species. During the course of this work, nine additional species were found, mostly Colombian (Román-P. et al. 2015, 2016). Also, the related genus Elaphopsocoides Román-P., García Aldrete and González 2014, including two Colombian species was described. The genus Chaetoblaste García Aldrete and Román-P. (2015), presently including six Colombian species was also erected. The genus Steleops Enderlein, previously unknown in Colombia, includes now 18 species, some of them already described in the course of this work (see González et al. 2011a, b).

The family Myopsocidae had not been recorded before in Colombia. To date, within the frame of this project we have found 29 species in the genera Lichenomima and Myopsocus.

Up to 2004, only the genera Graphocaecilius, Tricholachesilla, Hemicaecilius and Lachesilla, with a total of 12 species, were known in the family Lachesillidae in Colombia. As a result of this work, the family is now represented in Colombia by 16 genera and 103 species. The genera Acantholachesilla, Anomolachesilla, Dagualachesilla and Dagualachesilloides were erected within the context of this work (García Aldrete et al. 2012, 2013, 2014). The genus Waoraniella, that had been considered as endemic to the Amazon Basin (García Aldrete and Mockford, 2011) was found above $1800 \mathrm{~m}$ in the central and western Andean Cordilleras (Saenz et al., 2018)

The species here recorded were collected in 19 of the 33 Colombian Departments, as follows: Valle del Cauca (252), Putumayo (104), Caquetá (69), Risaralda (65), Cauca (64), Huila (64), Cundinamarca (44), Norte de Santander (42), Amazonas (38), Magdalena (34), Boyacá (23), Meta (20), Chocó (17), Caldas (10), Nariño (8), Vaupés (8), Tolima (5), Quindío (3), and Antioquia (2). There are not records of 14 departments, and some have been poorly sampled, such as the last five in the list above. Then it would be reasonable to asume that the number of Colombian species could reach at least 1000 .

\section{ACKNOWLEDGMENTS}

We wish to thank Colciencias for a grant to RGO, NSG and ANGA (Project: "Revisión Taxonómica y Endemismos de los Psócidos (Psocodea: 'Psocoptera') de Parques Naturales de Colombia", Code 1106-65944236). We also thank Claudia Alejandra Medina, of the Alexander von Humboldt Institute, for giving us access to the Psocoptera in its collections, Libardo Suárez Fonseca, Orlando Benítez, Luis González and Iván Mojica Vera of the National Natural Park Tamá, Nianza Del Carmen
Angulo and Octavio Betancur of the NNP Los Katios, Ítalo Rodríguez and Gustavo Adolfo Herrera Suárez of the NNP Cueva de Los Guácharos, Juan Iván Sanchez, Claudia Isabel Acevedo Bueno, Rodrigo Sarria, of the NNP Munchique, for giving logistic support and assistance in the areas of sampling, María Girleza Ramírez and Gloria Teresita Serna, of the Otún-Quimbaya Flora and Fauna Sanctuary, Mélida Cruz and Antonio Molina, of the El Encanto Nature Reserve, Amparo Bubú of the Pericos Nature Reserve, Rubén Luna and Svanhild Büch of the Meremberg Nature Reserve, The Autonomous Corporation of Risaralda, and Ovidio Ledesma, from Planes de San Rafael (Risaralda). ANGA thanks Instituto de Biología, Universidad Nacional Autónoma de México, for continuous research support. RGO and NSC thank Departamento de Biología, Facultad de Ciencias Naturales y Exactas, and Vicerrectoría de Investigaciones, Universidad del Valle, Santiago de Cali, Colombia, for research support.

\section{LITERATURE CITED}

Badonnel, A. 1986. Psocoptères de Colombie (Insecta, Psocoptera). Spixiana 9(2): 179-223.

Calderón-Martínez, N., R. González-Obando \& A. N. García Aldrete. 2014. Descriptions and records of Cladiopsocidae and Dolabellopsocidae (Insecta: Psocodea: 'Psocoptera') from Valle del Cauca and National Natural Park Gorgona, Colombia. Zootaxa 3889 (1), 1-30.

García Aldrete, A. N. 2012. A new genus of Epipsocidae (Psocodea: 'Psocoptera'), from Valle del Cauca, and a re-appraisal of Goja Navás, 1927. Caldasia 34(1): 247256.

García Aldrete, A. N. 2014. Biodiversidad de Psocoptera (Insecta: Psocodea) en México. Revista Mexicana de Biodiversidad Suplemento 85 (S): 252-256.

García Aldrete, A. N. \& E. L. Mockford. 2009. A list of Psocoptera (Insecta: Psocodea) from Brazil. Revista Mexicana de Biodiversidad 80: 665-673.

García Aldrete, A. N. \& E. L. Mockford. 2011. A new species of Waoraniella (Psocodea: 'Psocoptera': Lachesillidae: Eolachesillinae) from the Reserva Florestal Ducke, Amazonas, Brazil. Acta Zoológica Mexicana (n.s.) 27(1): 123-127.

García Aldrete, A. N. \& C. Román-P. 2015. A new neotropical genus of Blastini (Psocodea: 'Psocoptera': Psocidae: Amphigerontiinae). Zootaxa 4000 (3): 392396.

García Aldrete, A. N., R. González Obando \& N. S. Carrejo. 2011a. A new Loneura from Colombia, and Colombian records of L. mirandaensis García Aldrete, and Loneuroides venezolanus García Aldrete (Psocodea: 'Psocoptera': Ptiloneuridae). Dugesiana 18 (1): 35-37.

García Aldrete, A. N., R. González Obando \& F. A. Sarria Sarria. 2011b. Three new species of Loneura (Psocodea:'Psocoptera': Ptiloneuridae) from Gorgona Island, Cauca, Colombia, with a new infrageneric 
classification. Zootaxa (3050): 55-62.

García Aldrete, A. N. \& E. L. Mockford. 2012. Capítulo 30. Psocoptera Shipley, 1904. P. 424- 437. In: Insetos do Brasil. Diversidade e taxonomia. J. A. Rafael, G. A. R. Melo, C. J. B. de Carvalho, S. A. Casari, and R. Constantino (Editores). Ribeirao Preto: Holos Editora.

García Aldrete, A. N., R. González Obando \& N. S. Carrejo. 2012. A new genus of Lachesillidae (Psocoptera: Eolachesillinae: Graphocaeciliini) from Colombia. Dugesiana 19 (2): 91-97.

García Aldrete, A. N., R. González Obando \& N. S. Carrejo 2012 a. Four new species of Hemicaecilius Enderlein (Lachesillidae: Psocoptera) from Valle del Cauca, Colombia. Zootaxa 3455: 49-60.

García Aldrete, A. N., J. A. Mendivil Nieto \& R. GonzálezObando. 2012 b. A pair of new sister species of Loneura (Psocodea, 'Psocoptera', Ptiloneuridae) from Valle del Cauca, Colombia, representing a new infrageneric group. ZooKeys 168: 65-76.

García Aldrete, A. N., R. González Obando \& N. S. Carrejo. 2013a. New genera of Lachesillidae (Psocodea: 'Psocoptera': Eolachesillinae: Graphocaeciliini) from Valle del Cauca, Colombia. Zootaxa 3647(4): 555-566.

García Aldrete, A. N., R. González Obando, N. S. Carrejo, J. A. Mendivil \& N. R. Calderón. 2013b. Epipsocetae (Psocodea: 'Psocoptera') from Valle del Cauca and NNP Gorgona, Colombia. Dugesiana 20 (2): 157-172.

García Aldrete, A. N., R. González Obando \& N. S. Carrejo. 2013 c. A new species of Euplocania Enderlein (Psocodea: 'Psocoptera': Ptiloneuridae), from Magdalena, Colombia, with a proposed classification of the genus. Dugesiana 20 (2): 149-156.

García Aldrete, A. N., R. González Obando \& C. Saldaña. 2013. Two new species of Lachesilla of Pedicularia group (Psocodea: 'Psocoptera'), from Valle del Cauca, Colombia. Revista Colombiana de Entomología 39 (2): 237-242.

García Aldrete, A. N., O. Saenz-Manchola \& R. González-Obando. 2014. Acantholachesilla gen. n. (Psocodea:'Psocoptera': Lachesillidae: Eolachesillinae: Graphocaeciliini) from Valle del Cauca, Colombia. Zootaxa 3821 (5): 567-574.

García Aldrete, A. N., R. González Obando \& N. S. Carrejo. 2016. New species of Loneuroides García Aldrete (Psocodea: 'Psocoptera': Ptiloneuridae) from Colombia, and description of the male $L$. venezolanus García Aldrete. Zootaxa 4093 (1): 103-117.

García Aldrete, A. N., J. A. Mendivil Nieto \& R. GonzálezObando. 2015. Two new monotypic genera of Epipsocidae (Psocodea: 'Psocoptera': Psocomorpha) from Valle del Cauca, Colombia. Dugesiana 22(2): 163170.

García Aldrete, A. N., R. González, A. Arenas-Clavijo \& R. Lopes-Ferreira. 2016. Rediscovery of Antilopsocus nadleri Gurney, 1965 (Psocodea, Troctomorpha, Pachytroctidae) in South America. Check List 12(5),
1979: 1-3.

González Obando, R., A. N. García Aldrete \& N. S. Carrejo. 2011a. A new species of Steleops Enderlein, and a Colombian record of S. pulcher New (Psocodea: 'Psocoptera': Psocidae). Zootaxa (2735): 23-27.

González, R., A. N. García Aldrete \& N. S. Carrejo 2011b. New species of Steleops (Psocodea: 'Psocoptera': Psocidae) from Brazil, Colombia, Mexico and Peru. Zootaxa 3139: 28-54.

González, R., C. L. Saldaña \& A. N. García Aldrete. 2013. Lachesillidae (Psocodea: 'Psocoptera') de Valle del Cauca y NNP Gorgona, Colombia. Boletín del Museo de Entomología de la Universidad del Valle 14 (1):5169.

González-Obando, R., A. N. García Aldrete \& N. S. CarrejoGironza. 2015. New species of Euplocania Enderlein (Psocodea: 'Psocoptera': Psocomorpha: Ptiloneuridae) from Colombia. Zootaxa 4033 (4): 507-528.

González-Obando, R., A. N. García Aldrete \& N. S. Carrejo. 2016. New species of Cladiopsocus Roesler (Psocodea: 'Psocoptera': Psocomorpha: Cladiopsocidae) from the Colombian Amazonia. Zootaxa 4127 (1): 060-080.

González-Obando, R., N. Carrejo-Gironza \& A. N. García Aldrete. 2017a. New species of Colombian Triplocania Roesler (Psocodea: 'Psocoptera': Ptiloneuridae). Monograph. Zootaxa 4336 (1): 001-113.

González-Obando, R., A. N. García Aldrete \& N. S. Carrejo. 2017b. Five new species of the genus Euplocania Enderlein (Psocodea, 'Psocoptera', Psocomorpha, Ptiloneuridae) from Colombia. ZooKeys 711: 81-101.

González-Obando, R., A. N. García Aldrete, \& N. Carrejo. 2018. New species and records of Cladiopsocus Roesler (Psocodea: 'Psocoptera': Psocomorpha: Cladiopsocidae) from Colombia. Zootaxa 4378 (2): 191-223.

Lienhard, C. and C. N. Smithers. 2002. Psocoptera (Insecta). World catalogue and bibliography. Instrumenta Biodiversitatis V. Musèum d'histoire naturelle, Genève.

Lienhard, C. 2004. Worldwide country checklists of Psocoptera species [based on the World Catalogue by Lienhard and Smithers, 2002].

Mendivil Nieto, J. A., R. González Obando \& A. N. García Aldrete. 2015. Two new species of Edmockfordia García Aldrete (Psocodea, 'Psocoptera', Epipsocidae), from Valle del Cauca, Colombia, and description of the female E. chiquibulensis García Aldrete. ZooKeys 503: $45-54$.

Mendivil Nieto, J. A., A. N. García Aldrete \& R. González Obando R. 2017. Seven new species of Loneura Navás (Insecta: Psocodea: 'Psocoptera': Ptiloneuridae) from Valle del Cauca, Colombia. Zootaxa 4227 (4): 495-523.

Mockford, E. L. \& D. M. Sullivan. 1986. Systematics of the graphocaeciliine psocids with a proposed higher classification of the family Lachesillidae (Psocoptera). Transactions of the American Entomological Society 112 (1): 1-80. 
Román-P., C., A. N. García Aldrete \& R. González Obando. 2014. Elaphopsocoides, a new genus of Psocidae (Psocodea. 'Psocoptera') from Valle del Cauca, Colombia. Zootaxa 3873 (1): 93-100.

Román-Palacios, C., A. N. García Aldrete \& R. González Obando. 2016. Historical biogeography of Thyrsophorini psocids and description of a new neotropical species of Thyrsopsocopsis (Psocodea: Psocomorpha: Psocidae). European Journal of Taxonomy 194: 1-16.

Román-Palacios, C., R. González Obando \& A. N. García Aldrete. 2015. The genus Elaphopsocus Roesler (Psocodea: Psocoptera: Psocidae) with six new species from Brazil and Colombia. Zootaxa 3957 (2): 151-168.

Román-Palacios, C., R. González \& A. N. García Aldrete. 2016. Uncovering the diversity of the neotropical genus Elaphopsocus ('Psocoptera': Psocidae: Amphigerontiinae): from one to ten species. Zootaxa 4162 (2): 373-380.

Saenz, O. F, R. González Obando \& A. N. García Aldrete. 2014. Ectopsocidae (Psocodea: 'Psocoptera') from Valle del Cauca and NNP Gorgona, Colombia. Zootaxa 3786 (5): 523-540.

Saenz, O. F., A. N. García Aldrete \& R. González Obando. 2015. New species of Lachesilla Westwood in the pedicularia group (Psocodea: 'Psocoptera': Lachesillidae) from the Colombian Amazonia. Zootaxa 3915 (4): 510-520.

Saenz Manchola, O. F., R. González Obando \& A. N. García Aldrete. 2018. The genus Waoraniella García Aldrete (Lachesillidae: Eolachesillinae: Waoraniellini) in Colombia: Two new species from the Andean region. Zootaxa 4374 (2): 283-293.
Sandoval-Arango, S., R. González-Obando \& A. N. García Aldrete. 2016. New species of Graphocaecilius Enderlein (Psocodea: 'Psocoptera': Lachesillidae) from Colombia. Zootaxa 4147 (4): 421-432.

Sarria Sarria, F. A. 2011. Psocópteros (Insecta: 'Psocoptera') de cuatro biotopos del Parque Nacional Natural Gorgona (Cauca, Colombia). Tesis de Biólogo. Universidad del Valle, Facultad de Ciencias Naturales y Exactas, Programa Académico de Biología. Santiago de Cali, Colombia. 63 p.

Sarria-S., F., R. González Obando \& A. N. García Aldrete. 2014a. Psocoptera (Insecta: Psocodea) from the National Natural Park Gorgona, Cauca, Colombia, Revista de Biología Tropical 62: 243-256.

Sarria-S., F., R. González Obando \& A. N. García Aldrete. 2014b. Diversidad de Psocoptera (Insecta: Psocodea) en el Parque Nacional Natural Gorgona (Océano Pacífico, Colombia). Revista de Biología Tropical 62: 257-264.

Silva Neto, A. M., F. Bravo \& A. N. García Aldrete. 2014. Capítulo 10. Psocídeos (Psocodea: 'Psocoptera') do Semiárido: uma diversidade subestimada. Pp. 117-126. En: Bravo, F. \& Calor, A. (Organizadores). Artrópodes do Semiárido. Biodiversidade e Conservaçao. Feira de Santana. Printmídia.

Silva Neto, A. M. \& A. N. García Aldrete. 2016. Psocoptera in Catálogo Taxonómico da fauna do Brasil. PNUD.

Zhang, Z.-Q. 2013. Phylum Arthropoda. In: Zhang, Z.-Q. 2013. (Ed.). Animal Biodiversity: An outline of higher level classification and survey of taxonomic richness (Addenda 2013). Zootaxa 3703 (1): 1-82. 
Table 1. Families and number of genera and species per family of Psocodea: 'Psocoptera' recorded in Colombia.

\begin{tabular}{|c|c|c|c|}
\hline SUBORDER & FAMILY & No. GENERA & No. SPECIES \\
\hline \multirow{3}{*}{ TROGIOMORPHA } & Lepidopsocidae & 6 & 7 \\
\hline & Psoquillidae & 2 & 2 \\
\hline & Psyllipsocidae & 2 & 2 \\
\hline \multirow{7}{*}{ TROCTOMORPHA } & Amphientomidae & 3 & 3 \\
\hline & Compsocidae & 1 & 1 \\
\hline & Electrentomidae & 1 & 2 \\
\hline & Liposcelididae & 3 & 13 \\
\hline & Musapsocidae & 1 & 2 \\
\hline & Pachytroctidae & 4 & 5 \\
\hline & Troctopsocidae & 2 & 2 \\
\hline \multirow{19}{*}{ PSOCOMORPHA } & Amphipsocidae & 1 & 3 \\
\hline & Archipsocidae & 2 & 6 \\
\hline & Asiopsocidae & 1 & 2 \\
\hline & Caeciliusidae & 3 & 13 \\
\hline & Cladiopsocidae & 1 & 27 \\
\hline & Dasydemellidae & 2 & 4 \\
\hline & Dolabellopsocidae & 2 & 10 \\
\hline & Ectopsocidae & 2 & 16 \\
\hline & Epipsocidae & 13 & 148 \\
\hline & Hemipsocidae & 1 & 2 \\
\hline & Lachesillidae & 16 & 103 \\
\hline & Myopsocidae & 2 & 29 \\
\hline & Peripsocidae & 2 & 13 \\
\hline & Philotarsidae & 3 & 5 \\
\hline & Pseudocaecilidae & 4 & 5 \\
\hline & Psocidae. & 20 & 153 \\
\hline & Ptiloneuridae & 7 & 141 \\
\hline & Stenopsocidae & 1 & 1 \\
\hline & TOTAL & 108 & 720 \\
\hline
\end{tabular}



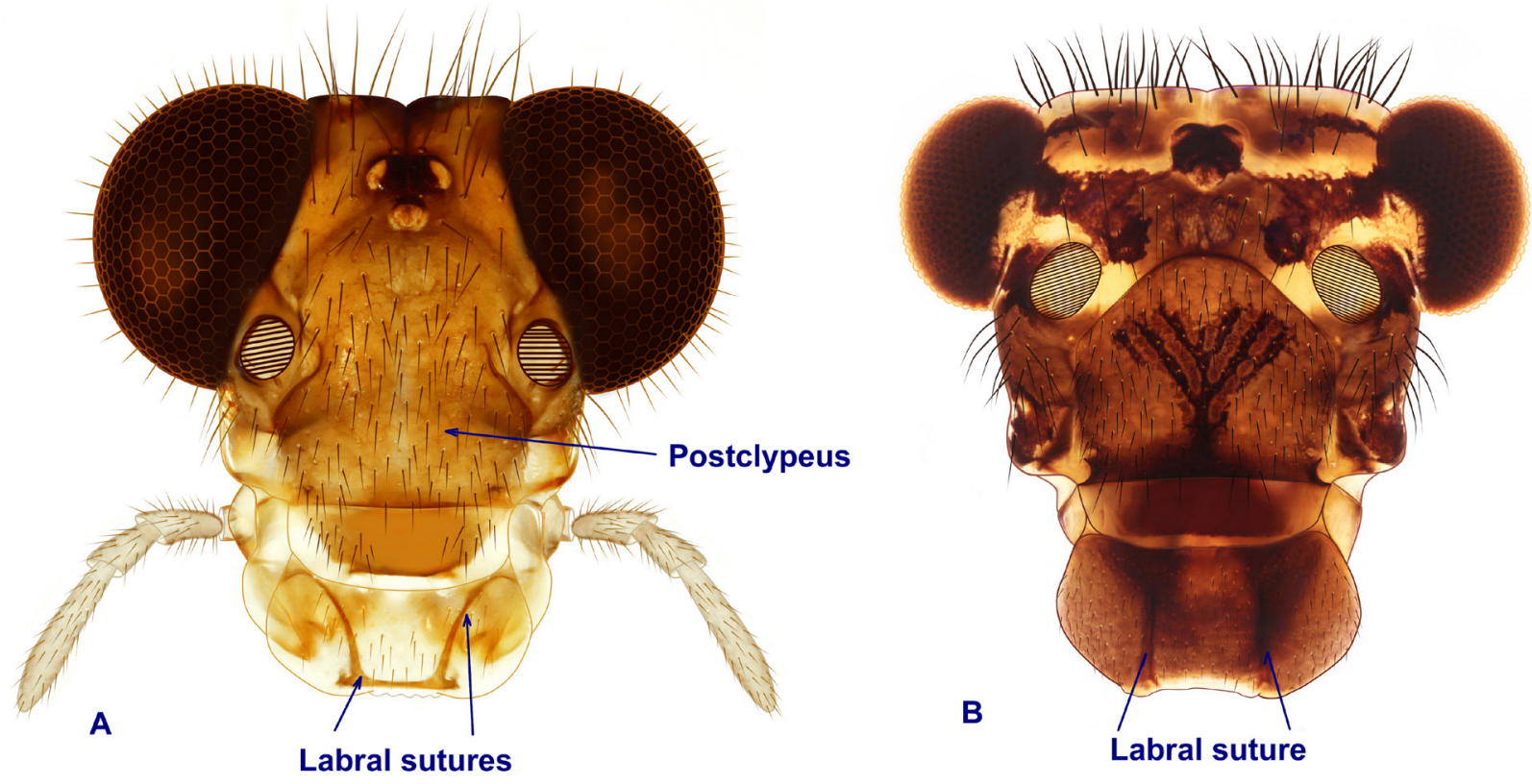

Figure 1. Psocomorpha. Front view of head. A. Epipsocetae. Cladiopsocidae. Cladiopsocus (Antennae removed). B. Ptiloneuridae. Triplocania (Antennae and maxillary palps removed).

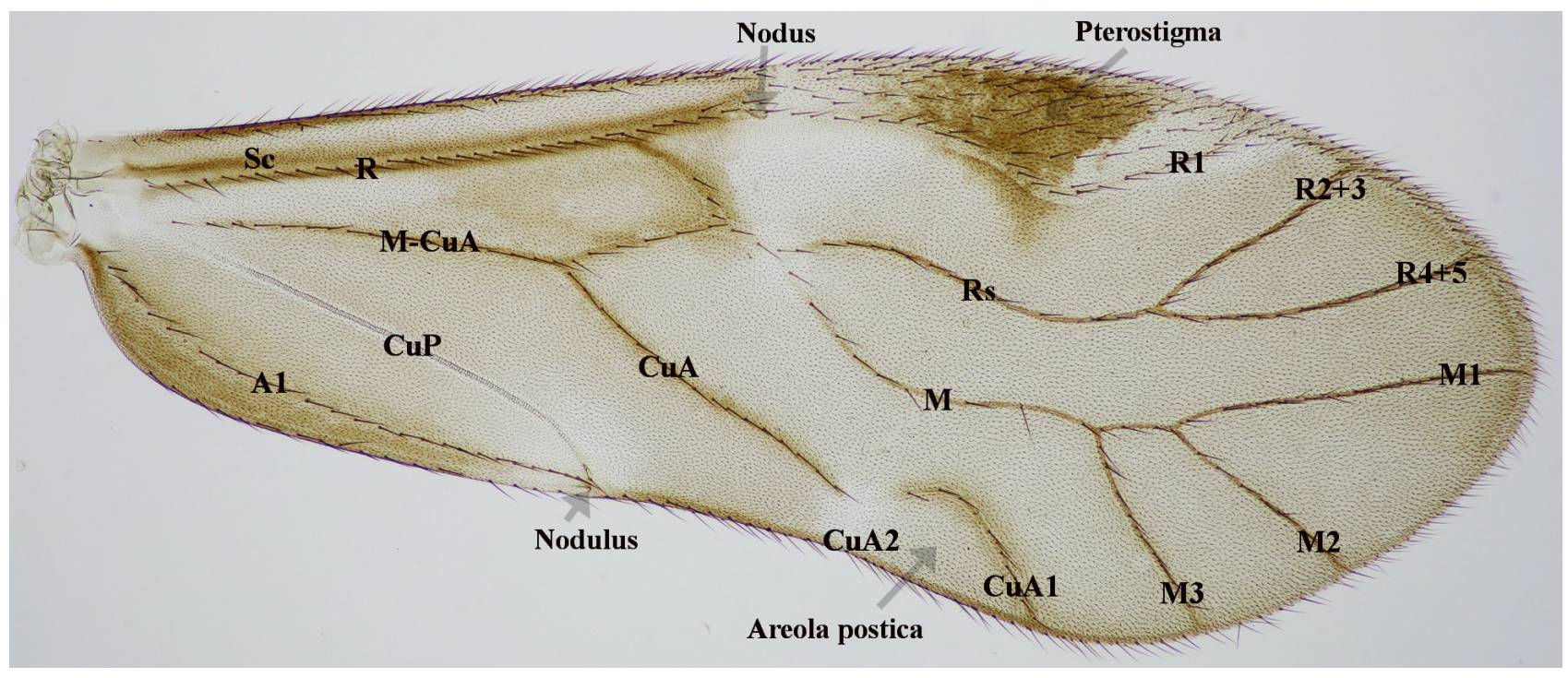

Figure 2. Forewing. Psocomorpha. Caeciliusidae. Valenzuela. 

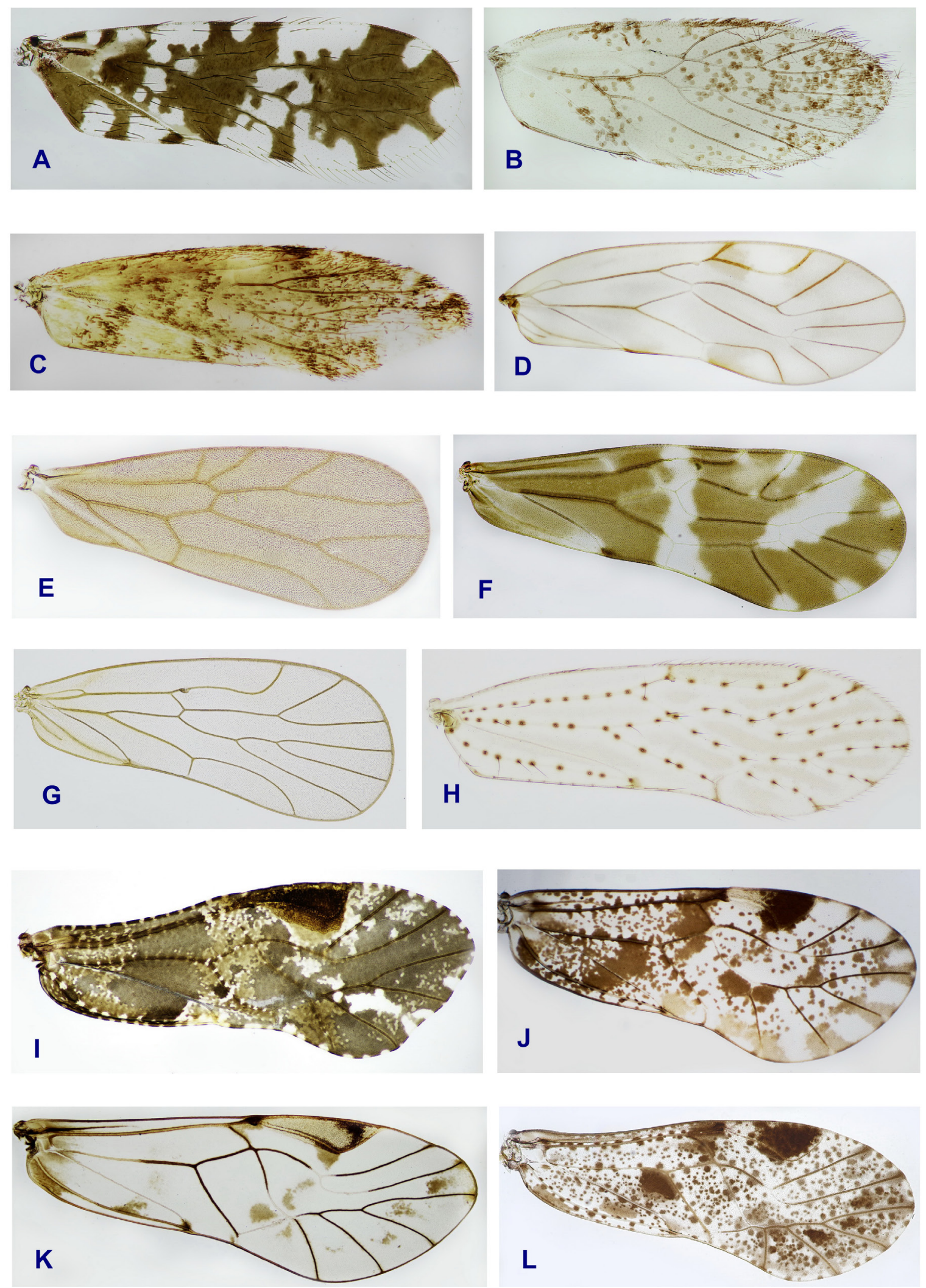

Figure 3. Forewings. Psocodea: 'Psocoptera'. A. Psoquillidae. Psoquilla. B. Lepidopsocidae. Soa. C. Amphientomidae. Amphientomum. D. Electrentomidae. Epitroctes. E. Pachytroctidae. Peritroctes. F. Troctopsocidae. Troctopsocopsis. G. Musapsocidae. Musapsocus. H. Hemipsocidae. Hemipsocus. I. Myopsocidae. Myopsocus. J, K, L. Psocidae. J. Elaphopsocus. K. Metylophorus. L. Trichadenotecnum. 

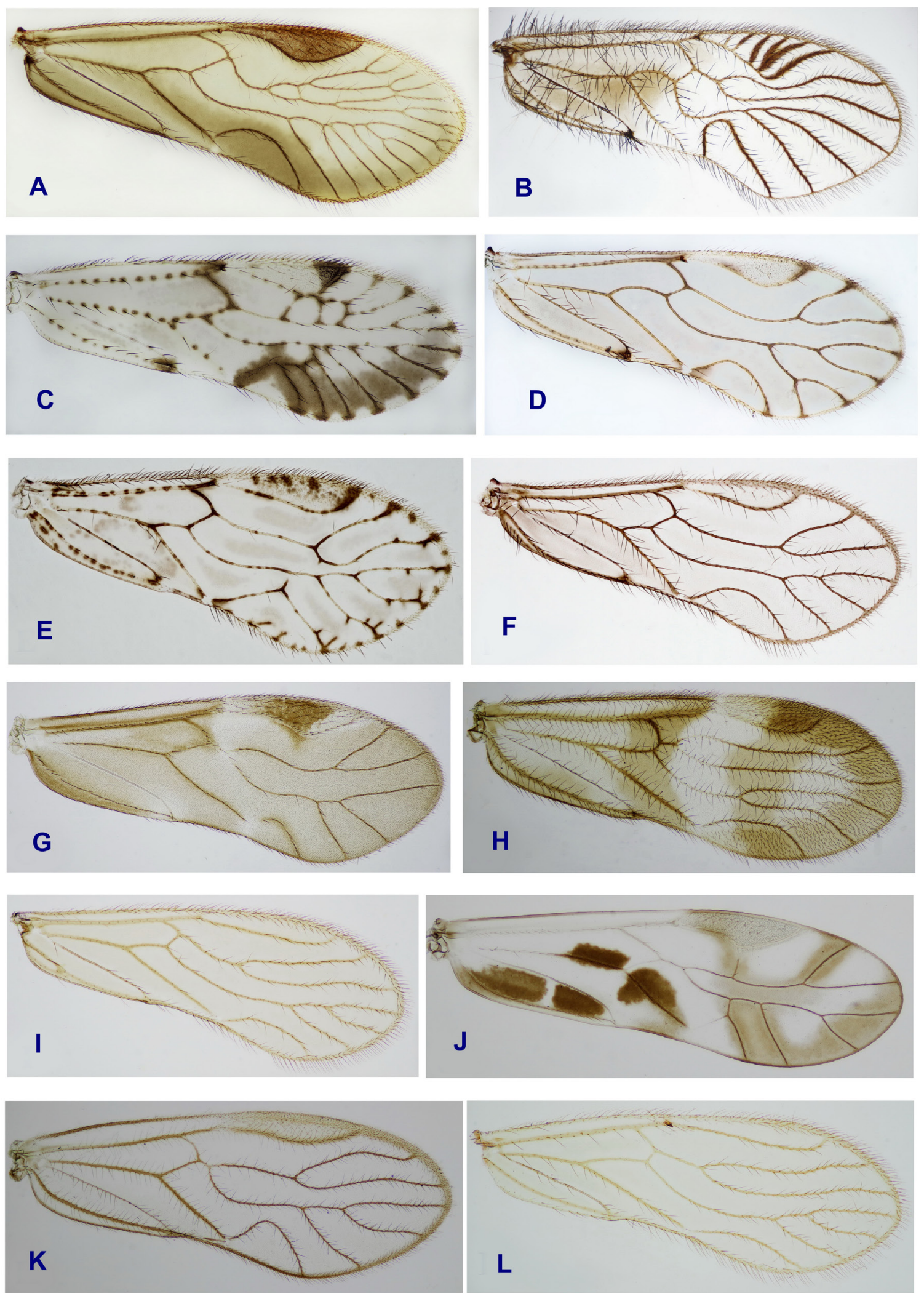

Figure 4. Forewings. Psocodea: 'Psocoptera'. A-D. Epipsocidae. A. Goja. B. Neurostigma. C. Epipsocus. D. Edmockfordia. E-F. Cladiopsocidae: E. Cladiopsocus. F. Cladiopsocus. G. Caeciliusidae. Valenzuela. H. Amphipsocidae. Polypsocus. I. Dolabellopsocidae. Isthmopsocus. J. Stenopsocidae. Graphopsocus. K. Dasydemellidae. Dasydemella. L. Dolabellopsocidae. Dolabellopsocus. 

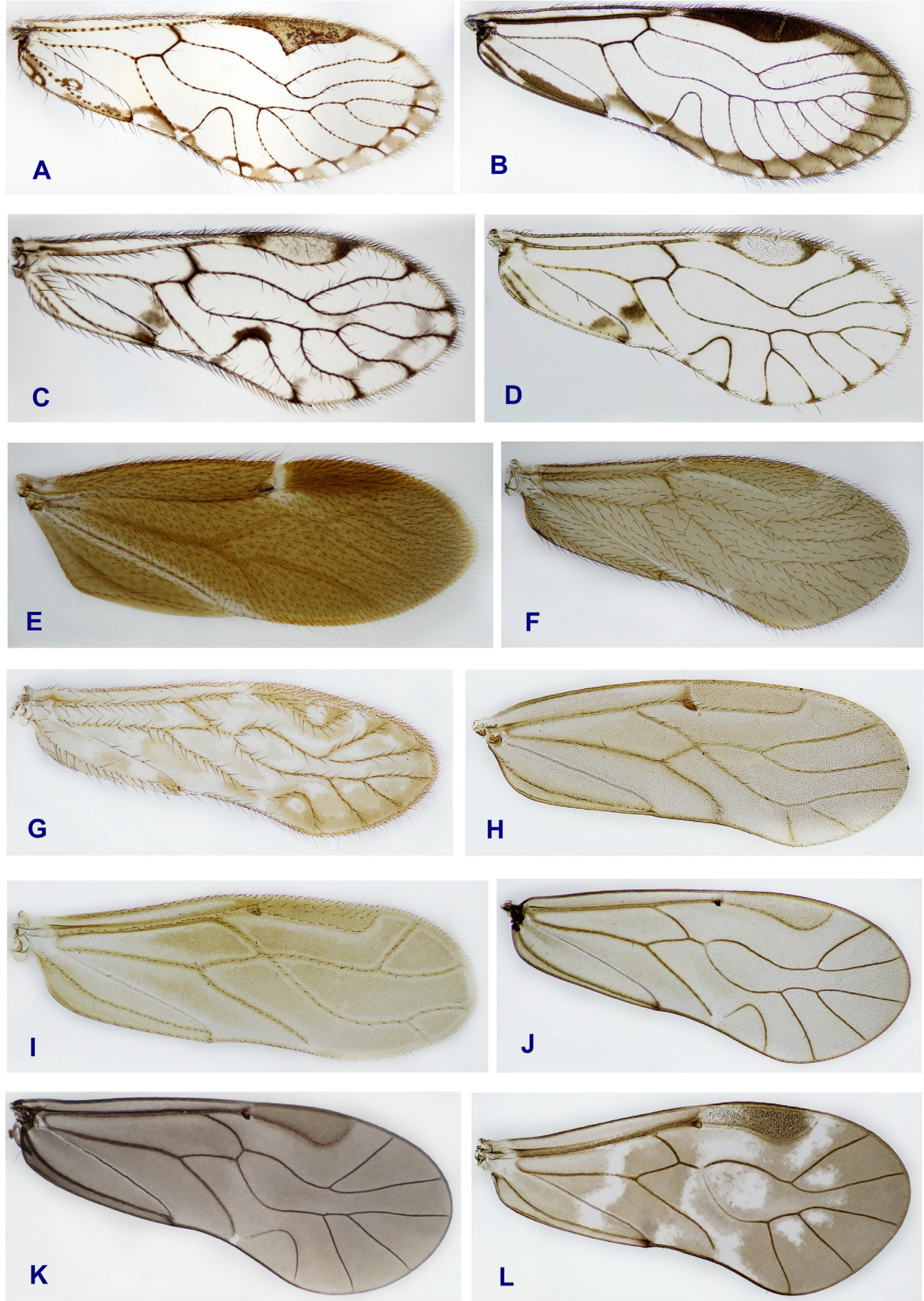

Figure 5. Forewings. Psocodea: 'Psocoptera'. A-D. Ptiloneuridae. A. Loneuroides. B. Loneura. C. Triplocania. D. Euplocania. E. Archipsocidae. Archipsocus. F. Pseudocaeciliidae. Scytopsocus. G. Philotarsidae. Aaroniella. H. Ectopsocidae. Ectopsocus. I. Asiopsocidae. Notiopsocus. J, K. Lachesillidae. J. Prolachesilla. K. Hemicaecilius. L. Peripsocidae. Peripsocus. 